\title{
A CAPACITABILITY THEOREM IN MEASURABLE GAMBLING THEORY
}

\author{
A. MAITRA, R. PURVES, AND W. SUDDERTH
}

\begin{abstract}
A player in a measurable gambling house $\Gamma$ defined on a Polish state space $X$ has available, for each $x \in X$, the collection $\Sigma(x)$ of possible distributions $\sigma$ for the stochastic process $x_{1}, x_{2}, \ldots$ of future states. If the object is to control the process so that it will lie in an analytic subset $A$ of $H=X \times X \times \cdots$, then the player's optimal reward is
\end{abstract}

$$
M(A)(x)=\sup \{\sigma(A): \sigma \in \Sigma(x)\} .
$$

The operator $M(\cdot)(x)$ is shown to be regular in the sense that

$$
M(A)(x)=\inf M(\{\tau<\infty\})(x),
$$

where the infimum is over Borel stopping times $\tau$ such that $A \subseteq\{\tau<\infty\}$. A consequence of this regularity property is that the value of $M(A)(x)$ is unchanged if, as in the gambling theory of Dubins and Savage, the player is allowed to use nonmeasurable strategies. This last result is seen to hold for bounded, Borel measurable payoff functions including that of Dubins and Savage.

\section{INTRODUCTION}

Let $X$ be a nonempty Borel subset of a Polish space and let $P(X)$ be the collection of countably additive probability measures defined on the Borel subsets of $X$. Give $P(X)$ its usual weak topology so that it too has the structure of a Borel subset of a Polish space (see Parthasarathy [16] for information about the weak topology on $P(X)$ ). An analytic gambling house $\Gamma$ is a mapping which assigns to each $x \in X$ a nonempty subset $\Gamma(x)$ of $P(X)$ in such a way that the set

$$
\Gamma=\{(x, \gamma) \in X \times P(X): \gamma \in \Gamma(x)\}
$$

is analytic. Starting at some initial state $x \in X$, a player in the house $\Gamma$ chooses a measurable strategy $\sigma$ available at $x$, which means a sequence $\sigma=$ $\left(\sigma_{0}, \sigma_{1}, \ldots\right)$, where $\sigma_{0} \in \Gamma(x)$ and, for $n=1, \sigma_{n}$ is a universally measurable mapping from $X^{n}$ to $P(X)$ such that $\sigma_{n}\left(x_{1}, x_{2}, \ldots, x_{n}\right) \in \Gamma\left(x_{n}\right)$ for every $\left(x_{1}, x_{2}, \ldots, x_{n}\right) \in X^{n}$. Every measurable strategy $\sigma$ determines a probability measure, also denoted by $\sigma$, on the Borel subsets of

$$
H=X \times X \times \cdots .
$$

Received by the editors May 16, 1990.

1980 Mathematics Subject Classification (1985 Revision). Primary 60G40, 93E20, 04A15, 28A12.

Key words and phrases. Measurable gambling, stochastic control, regularity, capacity, analytic sets, hyperarithmetic recursion.

The third author's research was supported by National Science Foundation Grant DMS-8801085. 
The probability measure $\sigma$ can be regarded as the distribution of the coordinate process $h=\left(h_{1}, h_{2}, \ldots\right)$, where $h_{1}$ has distribution $\sigma_{0}$ and $h_{n+1}$ has conditional distribution $\sigma_{n}\left(x_{1}, x_{2}, \ldots, x_{n}\right)$ given $h_{1}=x_{1}, h_{2}=x_{2}, \ldots, h_{n}=x_{n}$. For each $x \in X$, let $\Sigma(x)$ be the collection of measurable strategies available at $x$.

The optimal reward operator $M$ assigns to each bounded, universally measurable function $g: H \rightarrow \Re$ the function $M g$ defined on $X$ by

$$
(M g)(x)=\sup \left\{\left(\int g d \sigma: \sigma \in \Sigma(x)\right)\right\} .
$$

If $g$ is the indicator function of a universally measurable set $B$, we write $M(B)$ for $M g$. Thus, for fixed $x, M(\cdot)(x)$ is a set function. Regularity properties of this set function were studied in earlier papers $[12,13,19]$ and the major result of the present work is another such property which is analogous to the capacitability theorem of Choquet [2].

To state the result, it is convenient to introduce two topologies on $H$. Let $T_{1}$ be the product topology on $H$ when $X$ is assigned the topology under which it is a Borel subset of a Polish space, and let $T_{2}$ be the product topology on $H$ when $X$ has the discrete topology. The words "Borel," "analytic," "coanalytic," and "universally measurable," when used to qualify subsets of $H$, will refer to the topology $T_{1}$, while the words "closed," "open," "clopen," and " $G_{\delta}$," will refer to $T_{2}$. For a subset $E$ of $H$, define the function $M^{*}(E)$ on $X$ by

$$
M^{*}(E)(x)=\inf \{M(0)(x): E \subseteq 0 \text { and } 0 \text { is Borel, open }\} .
$$

Our main result is the following theorem.

Theorem 1.1. If $A$ is an analytic subset of $H$, then $M(A)=M^{*}(A)$.

This theorem was proved in [13] for the special case when $X$ is countable and also for the special case when $\Gamma(x)$ is finite for every $x$. For these cases, it was shown in [13] that $M^{*}(\cdot)(x)$ is a right-continuous (with respect to the topology $T_{1}$ ) capacity for each $x$. The result then follows from the capacitability theorem. It was also shown in [13] that $M^{*}(\cdot)(x)$ is not a capacity in general.

The proof of Theorem 1.1 will use ideas and techniques from the proof of the capacitability theorem, but also from gambling theory and effective descriptive set theory. The principal difficulty in proving Theorem 1.1 is in establishing the measurability of the function $M^{*}(A)(\cdot)$. It is already hard to do so when $A$ is a Borel, closed subset of $H$ (see [12] and remarks in [19]). We get around this problem by considering an effective surrogate of the function $M^{*}(A)(\cdot)$ and formulating an effective refinement of Theorem 1.1. This will involve the machinery of hyperarithmetic recursion, which will be explained in $\S 3$. Our proof of the effective refinement of Theorem 1.1 will proceed in stages. The first step will be to establish the result for effective Borel sets which are countable intersections of open sets in the topology $T_{2}$. This is done in $\S 5$. The next step, carried out in $\S 6$, is to extend the result to effective Borel sets which are countable unions of countable intersections of open sets. The final step, in $\S 7$, proves the result for effective analytic sets (Theorem 7.1). Theorem 1.1 is then deduced in $\S 8$.

We regard Theorem 1.1 as a fundamental approximation result in gambling theory as is the usual capacitability theorem in probability and measure theory. 
It will be applied in $\S 9$ to prove Theorem 1.2 , which answers an old question about the adequacy of measurable strategies.

Recall that the gambling theory of Dubins and Savage [4] takes place in a very general finitely additive framework in which a player is not restricted to measurable strategies. In the theory of Dubins and Savage, as extended by Purves and Sudderth [18], the probability $\sigma(A)$ is defined for every analytically measurable (that is, measurable with respect to the $\sigma$-field generated by analytic sets) set $A$ under every strategy $\sigma$, measurable or not. In consequence, $\int g d \sigma$ is defined for every bounded $g: H \rightarrow \mathfrak{R}$ which is upper analytic in the sense that $\{h \in H: g(h)>r\}$ is analytic for every real $r$. For such a payoff function $g$, the optimal reward to a player with initial state $x$ who is not restricted to measurable $\sigma$ is

$$
(\Gamma g)(x)=\sup \left\{\int g d \sigma: \sigma \text { available at } x\right\} .
$$

However, the player gains no advantage through the use of nonmeasurable $\sigma$ 's.

Theorem 1.2. If $g$ is bounded and upper analytic, then $\Gamma g=M g$.

This theorem generalizes Theorem 2.1 of Dubins et al. [5] which corresponds to the special case when $g(h)=\overline{\lim } u\left(h_{n}\right)$ for some bounded, upper analytic $u: X \rightarrow \mathfrak{R}$.

\section{Definitions AND NOtation}

$N$ will denote the set of positive integers, $\omega$ the set of natural numbers, and $\mathfrak{R}$ the set of real numbers. If $s, t$ are finite sequences of natural numbers, we write $|s|$ for the length of $s, s_{i}$ for the $i$ th coordinate of $s$ for $1 \leq i \leq|s|$, $s \supseteq t$ to denote that $s$ extends $t$, and $s t$ for the catenation of $s$ followed by $t$. If $\alpha \in \omega^{N}$ and $n \in N$, the finite sequence $(\alpha(1), \alpha(2), \ldots, \alpha(n))$ will be denoted by $\bar{\alpha}(n)$.

We use $X$ exclusively to denote the state space. It will always be a Borel subset of a Polish space. For $p \in X^{m}$ and $\left(x_{1}, x_{2}, \ldots, x_{n}\right) \in X^{n}, p\left(x_{1}, x_{2}, \ldots, x_{n}\right)$ or $p x_{1}, x_{2} \cdots x_{n}$ will denote the element of $X^{m+n}$ obtained by catenating $p$ and $\left(x_{1}, x_{2}, \ldots, x_{n}\right)$. The symbol $H$ will be reserved for $X^{N}$, the space of histories. For $h \in H$ and $i \in N, h_{i}$ will denote the $i$ th coordinate of $h$. If $h, h^{\prime} \in H$ and $n \in \omega$, we write $h \equiv{ }_{n} h^{\prime}$ if $h_{i}=h_{i}^{\prime}$ for $i=1,2, \ldots, n$. If $p \in X^{m}$ and $h \in H$, ph will be the element of $H$ obtained by catenating $p$ and $h$. For $k \in N, p_{k}$ will denote the function on $H$ to $X^{k}$ defined by $p_{k}(h)=\left(h_{1}, h_{2}, \ldots, h_{k}\right)$. If $B \subseteq H$ and $p \in X^{m}$, then $B p$ will denote the set of $h \in H$ such that $p h \in B$. Similarly, $B p_{k}$ will be the set-valued function on $H$ whose value at $h$ is the set $B p_{k}(h)$.

A mapping $\tau$ from $H$ to $N \cup\{\infty\}$ is called a stopping time if

$$
\tau(h)=n \in N \text { and } h^{\prime} \equiv_{n} h \quad \text { imply } \quad \tau\left(h^{\prime}\right)=n .
$$

A stop rule is an everywhere finite stopping time. A stopping time $\tau$ is Borel (universally) measurable if for each $n \in N$, the set $\{\tau \leq n\}$ is Borel (universally) measurable in $H$. If $\tau$ is a stopping time, then $h_{\tau}$ and $p_{\tau}$ are functions on the set $\{\tau<\infty\}$ whose values at $h$ are $h_{\tau(h)}$ and $p_{\tau(h)}(h)=$ $\left(h_{1}, h_{2}, \ldots, h_{\tau(h)}\right)$, respectively. Similarly, $B p_{\tau}$ is the set-valued function on 
$\{\tau<\infty\}$ whose value at $h$ is $B p_{\tau}(h)$. If $x \in X$, define $\tau[x]$ on $H$ by

$$
\tau[x](h)=\tau(x h)-1 \text {. }
$$

Then $\tau[x]$ is again a stopping time or identically equal to zero. We write $\tau\left[\left(x_{1}, x_{2}, \ldots, x_{n}\right)\right]$ or just $\tau\left[x_{1}, x_{2}, \ldots, x_{n}\right]$ for $\tau\left[x_{1}\right]\left[x_{2}\right] \cdots\left[x_{n}\right]$. If $k \in N$, define the stop rule $\tau \wedge k$ by

$$
(\tau \wedge k)(h)=\operatorname{minimum}\{\tau(h), k\} .
$$

Let $t$ be a stop rule. We say that a function $\phi$ on $H$ to a set $Y$ is determined by time $t$ if

$$
t(h)=k \& h \equiv{ }_{k} h^{\prime} \quad \text { imply } \quad \phi(h)=\phi\left(h^{\prime}\right) .
$$

A subset $K$ of $H$ is said to be determined by time $t$ just in case the indicator function of $K$ is determined by time $t$. It is easy to prove that a subset of $H$ is clopen in the topology $T_{2}$ iff it is determined by time $t$ for some stop rule $t$. A function $\psi$ on $H \times N$ to $Y$ is adapted if

$$
h \equiv{ }_{k} h^{\prime} \quad \text { implies } \quad \psi(h, k)=\psi\left(h^{\prime}, k\right) .
$$

Suppose $\sigma$ is a measurable strategy available at $x$ and $p \in X^{m}$. We define a measurable strategy $\sigma[p]$ available at $l(p)$, the last coordinate of $p$, as follows:

$$
(\sigma[p])_{0}=\sigma_{m}(p)
$$

and, for $n \geq 1$,

$$
(\sigma[p])_{n}\left(x_{1}, x_{2}, \ldots, x_{n}\right)=\sigma_{m+n}\left(p x_{1}, x_{2} \cdots x_{n}\right) .
$$

It is easy to verify that the measures (on $H$ induced by) $\sigma[p], p \in X_{m}$, are a version of the conditional $\sigma$-distribution of $\left(h_{m+1}, h_{m+2}, \ldots\right)$ given $\left(h_{1}, h_{2}, \ldots, h_{m}\right)=p$. If $\tau$ is a universally measurable stopping time, $\sigma\left[p_{\tau}\right]$ is a mapping on $\{\tau<\infty\}$ whose value at $h$ is $\sigma\left[p_{\tau}(h)\right]$.

By virtue of Lemma 2.2 in [11], we can fix a Borel measurable mapping $\nu: P(H) \times X \rightarrow P(H)$ such that for each $\mu \in P(H)$ and $x \in X, \nu(\mu, x)$ is a regular conditional $\mu$-distribution of $\left(h_{2}, h_{3}, \ldots\right)$ given $h_{1}=x$. For $\mu \in$ $P(H)$ and $x \in X$, we write $\mu[x]$ for $\nu(\mu, x)$; more generally, if $x_{1}, x_{2}, \ldots$, $x_{n} \in X$, we shall write $\mu\left[\left(x_{1}, x_{2}, \ldots, x_{n}\right)\right]$ or $\mu\left[x_{1}, x_{2}, \ldots, x_{n}\right]$ for $\mu\left[x_{1}\right]\left[x_{2}\right]$ $\cdots\left[x_{n}\right]$. If $\mu \in P(H), \mu_{0}$ will denote the marginal distribution of $\mu$ on the first coordinate of $H$. If $\tau$ is a universally measurable stopping time, $\mu\left[p_{\tau}\right]$ will denote the mapping from $\{\tau<\infty\}$ to $P(H)$ whose value at $h$ is $\mu\left[p_{\tau}(h)\right]$. We remark that this notation is in agreement with that of the previous paragraph in the following sense. If $\sigma$ is a measurable strategy and $\mu$ the probability measure induced on $H$ by $\sigma$, then, for almost all $(\mu) p \in X^{m}$, the measure induced by the strategy $\sigma[p]$ agrees with $\mu[p]$.

Suppose $\tau_{n}$ are universally measurable stopping times such that $\tau_{n}<\tau_{n+1}$ on $\left\{\tau_{n+1}<\infty\right\}, n \in N$. Assume that, for each $n \in N, \phi_{n}: H \times N \rightarrow P(H)$ is universally measurable and adapted. Let $\lambda \in P(H)$. Define, for each Borel subset $B$ of $X$,

$$
q_{0}(B)=\lambda_{0}(B)
$$


and, for $n \geq 1$,

$$
\begin{aligned}
& q_{n}\left(B \mid x_{1}, x_{2}, \ldots, x_{n}\right)=\left(\lambda\left[x_{1}, x_{2}, \ldots, x_{n}\right]\right)_{0}(B) \\
& \quad \text { if } n<\tau_{1}\left(\left(x_{1}, x_{2}, \ldots, x_{n}, x^{*}, x^{*}, \ldots\right)\right), \\
& =\left(\phi_{m}\left(\left(x_{1}, x_{2}, \ldots, x_{n}, x^{*}, x^{*}, \ldots\right), i\right)\left[x_{i+1}, x_{i+2}, \ldots, x_{n}\right]\right)_{0}(B) \\
& \quad \text { if } i=\tau_{m}\left(\left(x_{1}, x_{2}, \ldots, x_{n}, x^{*}, x^{*}, \ldots\right)\right) \\
& \leq n<\tau_{m+1}\left(\left(x_{1}, x_{2}, \ldots, x_{n}, x^{*}, x^{*}, \ldots\right)\right),
\end{aligned}
$$

where $x^{*}$ is an arbitrary fixed point of $X$ and the sequence $\left(x_{i+1}, x_{i+2}, \ldots, x_{n}\right)$ is to be regarded as the empty sequence if $n<i+1$. By [1, Proposition 7.45], there is a unique probability measure $\mu$ on the Borel subsets of $H$ such that $\mu_{0}=q_{0}$ and, for each $n \in N$, the $\mu$-conditional distribution of $h_{n+1}$ given $h_{1}=x_{1}, h_{2}=x_{2}, \ldots, h_{n}=x_{n}$ is $q_{n}\left(\cdot \mid x_{1}, x_{2}, \ldots, x_{n}\right)$ for almost all $(\mu)\left(x_{1}, x_{2}, \ldots, x_{n}\right) \in X^{n}$. We call $\mu$ the sequential composition of $\left(\lambda, \phi_{n}, \tau_{n}\right)$.

Recall from the previous section that if $\Gamma$ is an analytic gambling house then $\Sigma(x)$ is the set of measurable strategies available at $x$. Identifying measurable strategies with the probability measures they induce, we can consider $\Sigma(x)$ to be a set of probability measures on the Borel subsets of $H$. In order to establish the main result of this article, it will be convenient to work with these sets of probability measures rather than the gambling house $\Gamma$ which gives rise to them. We therefore isolate the properties of $\Sigma$ that will be needed and formulate the notion of a global gambling house.

A global gambling house $\Sigma$ is a mapping which assigns to each $x \in X$ a nonempty subset $\Sigma(x)$ of $P(H)$ in such a way that

(a) If $\mu \in \Sigma(x)$, then, for every $k \in N, \mu\left[x_{1}, x_{2}, \ldots, x_{k}\right] \in \Sigma\left(x_{k}\right)$ for almost all $(\mu)\left(x_{1}, x_{2}, \ldots, x_{k}\right) \in X^{k}$; and

(b) If $\tau_{n}$ are universally measurable stopping times such that $\tau_{n}<\tau_{n+1}$ on $\left\{\tau_{n+1}<\infty\right\}$ for $n \in N$, if, for each $n \in N, \phi_{n}: H \times N \rightarrow P(H)$ is universally measurable, adapted, and $\phi_{n}(h, k) \in \Sigma\left(h_{k}\right)$ for all $(h, k) \in H \times N$, and if $\lambda \in \Sigma(x)$, then the sequential composition of $\left(\lambda, \phi_{n}, \tau_{n}\right)$ belongs to $\Sigma(x)$.

We remark that if $\Gamma$ is an analytic gambling house and if $\Sigma(x)$ is the set of probability measures on $H$ induced by measurable strategies available at $x$ for each $x \in X$, then $\Sigma$ is a global gambling house. Though we shall have no use for it, we remark that the converse is also true; if $\Sigma$ is an analytic, global gambling house, then there is an analytic, "local" gambling house $\Gamma$ such that, for each $x \in X, \Sigma(x)$ is precisely the set of measures induced by measurable strategies available at $x$ in the house $\Gamma$.

\section{SOME EFFECTIVE DESCRIPTIVE SET THEORY}

Effective descriptive set theory takes place in Polish spaces which admit a smooth recursion theory. This is made precise in the next definition.

We say that a topological space $Z$ is $\Delta_{1}^{1}$-recursively presented if $Z$ admits a complete metric $d$ and a dense sequence $\left(r_{n}\right)_{n \in w}$ such that the relations

$$
d\left(r_{n}, r_{m}\right) \leq p / q+1 \quad \text { and } d\left(r_{n}, r_{m}\right)<p / q+1
$$

are $\Delta_{1}^{1}$ in $\omega^{4}$. Examples of such spaces are $2,2^{\omega}, \omega^{\omega},[0,1]^{\omega}$, etc. (see Moschovakis [15]). 
Suppose now that $Z_{1}$ and $Z_{2}$ are $\Delta_{1}^{1}$-recursively presented compact metric spaces. Then $Z_{1} \times Z_{2}$ and $P\left(Z_{1}\right)$, the set of probability measures on the Borel subsets of $Z_{1}$, are again $\Delta_{1}^{1}$-recursively presented compact metric spaces (Louveau [7, 9]).

In what follows, our terminology and notation, pertaining to concepts in effective descriptive set theory, are taken from Moschovakis [15].

Following Louveau $[8$, p. 13$]$, we say that the pair $(W, C)$ is a coding of Borel subsets of a $\Delta_{1}^{1}$-recursively presented compact metric space $Y$ if

(a) $W$ is a $\Pi_{1}^{1}$ subset of $\omega^{\omega} \times \omega$;

(b) $C$ is a $\Pi_{1}^{1}$ subset of $\omega^{\omega} \times \omega \times Y$;

(c) the set $\left\{(\alpha, n, y) \in \omega^{\omega} \times \omega \times Y:(\alpha, n) \in W \&(\alpha, n, y) \notin C\right\}$ is $\Pi_{1}^{1}$;

(d) for fixed $(\alpha, n) \in \omega^{\omega} \times \omega$, the section $C_{\alpha, n}=\{y \in Y:(\alpha, n, y) \in C\}$ is $\Delta_{1}^{1}(\alpha)$;

(e) If $P \subseteq Y$ is $\Delta_{1}^{1}(\alpha)$, then there is an $n$ such that $(\alpha, n) \in W$ and $P=C_{\alpha, n}$.

The rest of this section will be devoted to proving an effective separation theorem which will be needed in the sequel. In what follows and until further notice, $X$ will be $2^{\omega}$, the space of sequences of 0 's and 1's, and $H$ will be $X \times X \times \cdots$.

Fix a coding $(W, C)$ of Borel subsets of the $\Delta_{1}^{1}$-recursively presented compact metric space $H$. We will be interested in $(\alpha, n) \in W$ such that $C_{\alpha, n}$ is Borel and open. Define $\bar{W} \subseteq \omega^{\omega} \times \omega$ as follows:

$$
\begin{aligned}
(\alpha, n) \in \bar{W} \leftrightarrow(\alpha, n) \in W & \\
& \&(\forall h)\left[(\alpha, n, h) \notin C \text { or }(\exists m)\left(\forall h^{\prime}\right)\left(h^{\prime} \equiv_{m} h \rightarrow\left(\alpha, n, h^{\prime}\right) \in C\right)\right] .
\end{aligned}
$$

Clearly, $\bar{W}$ is $\Pi_{1}^{1}$, and if $(\alpha, n) \in W$, then $(\alpha, n) \in \bar{W}$ if and only if $C_{\alpha, n}$ is open. Set

$$
\bar{C}=C \cap(\bar{W} \times H) .
$$

Then

(i) $\bar{C}$ is $\Pi_{1}^{1}$;

(ii) the set $\left\{(\alpha, n, h) \in \omega^{\omega} \times \omega \times H:(\alpha, n) \in \bar{W} \&(\alpha, n, h) \notin \bar{C}\right\}$ is $\Pi_{1}^{1}$;

(iii) $P \subseteq H$ is $\Delta_{1}^{1}(\alpha)$ and open if and only if $P=\bar{C}_{\alpha, n}$ for some $(\alpha, n) \in$ $\bar{W}$.

Lemma 3.1. If $A$ and $B$ are $\Sigma_{1}^{1}$ subsets of $H$ such that $A$ can be separated from $B$ by an open set, then there is a $\Delta_{1}^{1}$-recursive stopping time $\tau$ such that

$$
A \subseteq\{\tau<\infty\} \subseteq B^{c} .
$$

Proof. Define $P \subseteq H \times N$ as follows:

$$
(h, n) \in P \leftrightarrow\left(\forall h^{\prime}\right)\left(h^{\prime} \equiv{ }_{n} h \rightarrow h^{\prime} \notin B\right) .
$$

Then $P$ is $\Pi_{1}^{1}$ and $(\forall h \in A)(\exists n)((h, n) \in P)$. So, by the Kreisel selection theorem [15, p. 203], there is a $\Delta_{1}^{1}$-recursive function $f: H \rightarrow N$ such that $(\forall h \in A)((h, f(h)) \in P)$. Let

$$
Q=\left\{(h, n) \in H \times N:\left(\exists h^{\prime} \in A\right)\left(h^{\prime} \equiv{ }_{n} h \& f\left(h^{\prime}\right)=n\right)\right\} .
$$


It follows that $Q$ is $\Sigma_{1}^{1}$ and $Q \subseteq P$. Next, we define $S \subseteq N \times \omega$ as follows:

$$
\begin{aligned}
(n, m) \in & S \leftrightarrow\left(x^{*}, m\right) \in \bar{W} \\
& \&(\forall h)\left(\forall h^{\prime}\right)\left(h \in \bar{C}_{x^{*}, m} \& h \equiv{ }_{n} h^{\prime} \rightarrow h^{\prime} \in \bar{C}_{x^{*}, m}\right) \\
& \&(\forall h)\left(h \in \bar{C}_{x^{*}, m} \rightarrow(h, n) \in P\right) \\
& \&(\forall h)\left((h, n) \in Q \rightarrow h \in \bar{C}_{x^{*}, m}\right),
\end{aligned}
$$

where $x^{*}$ is a fixed $\Delta_{1}^{1}$ point in $\omega^{\omega}$. Then $S$ is $\Pi_{1}^{1}$. Use the first principle of separation for $\Sigma_{1}^{1}$ sets [15, p. 204] to see that $(\forall n \in N)(\exists m)((n, m) \in$ $S)$. Invoke the Kreisel selection theorem one more time to get a $\Delta_{1}^{1}$-recursive function $g: N \rightarrow \omega$ such that $(\forall n \in N)((n, g(n)) \in S)$. Finally, define $\tau$ on $H$ as follows:

$$
\tau(h)= \begin{cases}\text { least } n \text { such that } h \in \bar{C}_{x^{*}, g(n)} & \text { if }(\exists n \in N)\left(h \in \bar{C}_{x^{*}, g(n)}\right), \\ \infty & \text { otherwise }\end{cases}
$$

Then it is easy to verify that $\tau$ satisfies the assertion of the lemma. laries.

As immediate consequences of the lemma, we have the following two corol-

Corollary 3.2. If $V$ is a $\Delta_{1}^{1}$, open subset of $H$, then there is a $\Delta_{1}^{1}$-recursive stopping time $\tau$ such that $V=\{\tau<\infty\}$.

Corollary 3.3. If $K$ is a $\Delta_{1}^{1}$, clopen subset of $H$, then there is a $\Delta_{1}^{1}$-recursive stop rule $\tau$ such that $K$ is determined by time $\tau$.

Classical (boldface) versions of the above results were proved in [10].

\section{A fundamental inequality}

Let $X=2^{\omega}, H=X \times X \times \cdots$, and $\Sigma \subseteq X \times P(H)$ be a global gambling house which is a $\Sigma_{1}^{1}$ subset of the $\Delta_{1}^{1}$-recursively presented compact metric space $X \times P(H)$.

Let $E \subseteq H$ be universally measurable and let $x \in X$. We can define

$$
M(E)(x)=\sup \{\mu(E): \mu \in \Sigma(x)\}, \quad x \in X .
$$

There should be no confusion with the symbol $M$ introduced in $\S 1$, where $\Sigma(x)$ denoted the set of measurable strategies available at $x$. Here we have no "local" gambling house $\Gamma$, but in case $\Sigma$ above had been induced by a "local" gambling house, the $M$ of (4.1) would have coincided with the same symbol of $\S 1$.

Next we define the effective surrogate $\bar{M}$ of the function $M^{*}$ of $\S 1$. However, $\bar{M}$ has to be defined simultaneously for a $\Sigma_{1}^{1}$ subset of $H$ and all its sections. So let $E$ be a $\Sigma_{1}^{1}$ subset of $H$ and let $x_{1}, x_{2}, \ldots, x_{k}, x \in 2^{\omega}$. We define

$$
\bar{M}\left(E ; x_{1}, x_{2}, \ldots, x_{k} ; x\right)=\inf \sup \{\mu(0): \mu \in \Sigma(x)\},
$$

where the inf is taken over all $\Delta_{1}^{1}\left(\left\langle x_{1}, x_{2}, \ldots, x_{k}, x\right\rangle\right)$, open sets $O$ containing $E x_{1} x_{2} \cdots x_{k}$. Here $\left\langle\alpha_{1}, \alpha_{2}, \ldots, \alpha_{k}\right\rangle$ is the member of $\omega^{\omega}$ which codes the finite sequence $\alpha_{1}, \alpha_{2}, \ldots, \alpha_{k}$ of members of $\omega^{\omega}$, as described in [15, p. 40]. Since $2^{\omega} \subseteq \omega^{\omega}$, the coding can be used for finite sequences of members 
of $2^{\omega}$ as well. We allow the finite sequence $x_{1}, x_{2}, \ldots, x_{k}$ to be the empty sequence in (4.2). In the sequel, the left side of (4.2) will be abbreviated by $\bar{M}\left(E x_{1} x_{2} \cdots x_{k}\right)(x)$. Similarly, if $\alpha \in \omega^{\omega}$ and $F$ is a $\Sigma_{1}^{1}(\alpha)$ subset of $H$, we define the relativization $\bar{M}^{\alpha}$ of $\bar{M}$ as follows:

$$
\bar{M}^{\alpha}\left(F ; x_{1}, x_{2}, \ldots, x_{k} ; x\right)=\inf \sup \{\mu(0): \mu \in \Sigma(x)\},
$$

where now the inf is taken over all $\Delta_{1}^{1}\left(\left\langle\alpha, x_{1}, x_{2}, \ldots, x_{k}, x\right\rangle\right)$, open sets $O$ containing $F x_{1} x_{2} \cdots x_{k}$. The left side of (4.3) will be abbreviated by

$$
\bar{M}^{\alpha}\left(F x_{1} x_{2} \cdots x_{k}\right)(x) \text {. }
$$

Now suppose that $F=E x_{1} x_{2} \cdots x_{k}$ for some $\Sigma_{1}^{1}$ subset $E$ of $H$ and $x_{1}, x_{2}, \ldots, x_{k} \in 2^{\omega}$. Set $\alpha=\left\langle x_{1}, x_{2}, \ldots, x_{k}\right\rangle$. Then, as is easy to verify,

$$
\bar{M}^{\alpha}(F ; x)=\bar{M}\left(E ; x_{1}, x_{2}, \ldots, x_{k} ; x\right) .
$$

We will use (4.4) without explicit mention in the sequel.

Lemma 4.1. If $E \subseteq H$ is $\Sigma_{1}^{1}$, then the set

$$
\left\{(h, k, x, a) \in H \times N \times X \times[0,1]: \bar{M}\left(E h_{1} h_{2} \cdots h_{k}\right)(x) \geq a\right\}
$$

is $\Sigma_{1}^{1}$.

Proof.

$$
\begin{aligned}
& \bar{M}\left(E h_{1} h_{2} \cdots h_{k}\right)(x) \geq a \\
& \leftrightarrow(\forall r)(\forall n)\left[r<a \&\left(\left\langle h_{1}, h_{2}, \ldots, h_{k}, x\right\rangle, n\right) \in \bar{W}\right. \\
& \quad \& E h_{1} h_{2} \cdots h_{k} \subseteq \bar{C}_{\left\langle h_{1}, h_{2}, \ldots, h_{k}, x\right\rangle, n} \\
& \\
& \quad \rightarrow(\exists \mu \in \Sigma(x))\left(\mu \left(\bar{C}_{\left.\left.\left.\left\langle h_{1}, h_{2}, \ldots, h_{k}, x\right\rangle, n\right)>r\right)\right],}\right.\right.
\end{aligned}
$$

where $r$ runs through the rationals in $[0,1]$, and we think of $2^{\omega}$ as as being embedded as a $\Pi_{1}^{0}$ subset of $\omega^{\omega}$. One sees easily by imitating the computation in the proof of Theorem 2.2.3(a) in Kechris [6] that the relation above is $\Sigma_{1}^{1}$.

Lemma 4.2. If $E \subseteq H$ is $\Sigma_{1}^{1}$ and $\tau$ is a $\Delta_{1}^{1}$-recursive stopping time on $H$, then the set

$$
\left\{(h, a) \in H \times[0,1]: \tau(h)<\infty \& \bar{M}\left(E p_{\tau}\right)\left(h_{\tau}\right) \geq a\right\}
$$

is $\Sigma_{1}^{1}$.

Proof.

$$
\begin{aligned}
\tau(h) & <\infty \& \bar{M}\left(E p_{\tau}\right)\left(h_{\tau}\right) \geq a \\
& \leftrightarrow(\exists k \in N)\left[\tau(h)=k \& \bar{M}\left(E h_{1} h_{2} \cdots h_{k}\right)\left(h_{k}\right) \geq a\right] .
\end{aligned}
$$

The above condition is $\Sigma_{1}^{1}$ by virtue of Lemma 4.1 .

Lemma 4.3. Let $E \subseteq H$ be $\Sigma_{1}^{1}$ and $\tau$ a $\Delta_{1}^{1}$-recursive stopping time on $H$. Fix $x_{0} \in X$ and $\varepsilon>0$ rational. Then there is a $\Delta_{1}^{1}\left(x_{0}\right)$-recursive function $g: H \rightarrow[0,1]$ such that

(i) $\tau(h)<\infty \Rightarrow g(h) \geq \bar{M}\left(E p_{\tau}\right)\left(h_{\tau}\right)$,

(ii) $\tau(h)=k<\infty \& h \equiv{ }_{k} h^{\prime} \Rightarrow g(h)=g\left(h^{\prime}\right)$, and

(iii) $\sup _{\mu \in \Sigma\left(x_{0}\right)} \int g(h) d \mu(h) \leq \sup _{\mu \in \Sigma\left(x_{0}\right)} \int_{\{\tau<\infty\}} \bar{M}\left(E p_{\tau}\right)\left(h_{\tau}\right) d \mu(h)+\varepsilon$. 
Proof. Let $X^{*}=\bigcup_{m \in \omega} X^{m}$. Now $X^{*}$ can be endowed with a $\Delta_{1}^{1}$-recursive presentation so it becomes a $\Delta_{1}^{1}$-recursively presented Polish space. Define $\psi: H \rightarrow X^{*}$ as follows:

$$
\psi(h)= \begin{cases}\left(h_{1}, h_{2}, \ldots, h_{\tau}\right) & \text { if } \tau(h)<\infty, \\ e & \text { if } \tau(h)=\infty,\end{cases}
$$

where $e$ is the empty sequence. Plainly, $\psi$ is $\Delta_{1}^{1}$-recursive.

Let $\widetilde{\boldsymbol{\Sigma}}\left(x_{0}\right)=\left\{\mu \psi^{-1}: \mu \in \Sigma\left(x_{0}\right)\right\}$, so that $\widetilde{\boldsymbol{\Sigma}}\left(x_{0}\right)$ is a $\Sigma_{1}^{1}\left(x_{0}\right)$ set in $P\left(X^{*}\right)$, and hence the set $\left\{\nu \times \lambda: \nu \in \widetilde{\Sigma}\left(x_{0}\right)\right\}$ is $\Sigma_{1}^{1}\left(x_{0}\right)$ in $P\left(X^{*} \times[0,1]\right)$, where $\lambda$ is Lebesgue measure. Finally, define $\Phi: X^{*} \rightarrow[0,1]$ by setting

$$
\Phi(p)= \begin{cases}\bar{M}(E p)(l(p)) & \text { if } p \in \bigcup_{m \geq 1} X^{m}, \\ 0 & \text { if } p=e,\end{cases}
$$

where $l(p)$ is the last coordinate of $p$. Let

$$
D=\left\{(p, a) \in X^{*} \times[0,1]: \Phi(p) \geq a\right\} .
$$

Note that by virtue of Lemma $4.1 D$ is $\Sigma_{1}^{1}\left(x_{0}\right)$. So, by Lemma 4.3 in [12], there is a $\Delta_{1}^{1}\left(x_{0}\right)$ set $B$ in $X^{*} \times[0,1]$ such that $D \subseteq B$ and

$$
\begin{aligned}
\sup _{\nu \in \widetilde{\Sigma}\left(x_{0}\right)}(\nu \times \lambda)(B) & \leq \sup _{\nu \in \widetilde{\Sigma}\left(x_{0}\right)}(\nu \times \lambda)(D)+\varepsilon=\sup _{\nu \in \widetilde{\Sigma}\left(x_{0}\right)} \int_{X^{*}} \Phi(p) d \nu(p)+\varepsilon \\
& =\sup _{\mu \in \Sigma\left(x_{0}\right)} \int_{\{\tau<\infty\}} \bar{M}\left(E p_{\tau}\right)\left(h_{\tau}\right) d \mu(h)+\varepsilon,
\end{aligned}
$$

where the last equality can be justified by first observing that $\bar{M}\left(E p_{\tau}\right)\left(h_{\tau}\right)=$ $\Phi(\psi(h))$ if $\tau(h)<\infty$ and then applying the change of variable theorem.

We now define $g: H \rightarrow[0,1]$ as follows:

$$
g(h)= \begin{cases}\lambda\left(B_{\psi(h)}\right) & \text { if } \tau(h)<\infty, \\ 0 & \text { otherwise }\end{cases}
$$

where $B_{\psi(h)}=\{a \in[0,1]:(\psi(h), a) \in B\}$. It is now easy to verify that $g$ satisfies the assertions of the lemma.

The next result is the fundamental inequality of the theory and can be regarded as one-half of the optimality equation.

Theorem 4.4. If $E \subseteq H$ is $\Sigma_{1}^{1}$ and $\tau$ is a $\Delta_{1}^{1}$-recursive stopping time on $H$ such that $E \subseteq\{\tau<\infty\}$, then

$$
\bar{M}(E)(x) \leq \sup _{\mu \in \Sigma(x)} \int_{\{\tau<\infty\}} \bar{M}\left(E p_{\tau}\right)\left(h_{\tau}\right) d \mu(h)
$$

for all $x \in X$.

Proof. Fix $x_{0} \in X$ and $\varepsilon>0$ rational. Apply Lemma 4.3 with $\varepsilon / 3$ to get a function $g$ satisfying the assertions of the lemma. Define $P \subseteq H \times \omega$ as follows:

$$
\begin{aligned}
(h, n) \in P \leftrightarrow(\exists k \in N)\left[\tau(h)=k \&\left(\left\langle h_{1}, h_{2}, \ldots, h_{k}\right\rangle, n\right) \in \bar{W}\right. \\
\quad \& E h_{1} h_{2} \cdots h_{k} \subseteq \bar{C}_{\left\langle h_{1}, h_{2}, \ldots, h_{k}\right\rangle, n} \\
\quad \&\left(\forall \mu \in \Sigma\left(h_{k}\right)\right)\left(\mu\left(\bar{C}_{\left\langle h_{1}, h_{2}, \ldots, h_{k}\right\rangle, n}\right)<g(h)+\varepsilon / 3\right] .
\end{aligned}
$$


Then $P$ is $\Pi_{1}^{1}\left(x_{0}\right)$ and one verifies easily that $(\forall h)[\tau(h)<\infty \rightarrow(\exists n)((h, n) \in$ $P)$ ]. An application of Kreisel's selection theorem yields a $\Delta_{1}^{1}\left(x_{0}\right)$-recursive function $f: H \rightarrow \omega$ such that $(h, f(h)) \in P$ whenever $\tau(h)<\infty$.

We now define $O \subseteq H$ by

$$
\begin{aligned}
& h \in O \leftrightarrow(\exists k \in N)\left[\tau(h)=k \&\left(\left\langle h_{1}, h_{2}, \ldots, h_{k}\right\rangle,\right.\right. \\
& \left.\left.\quad f\left(\left(h_{1}, h_{2}, \ldots, h_{k}, x^{*}, x^{*}, \ldots\right)\right),\left(h_{k+1}, h_{k+2}, \ldots\right)\right) \in \bar{C}\right],
\end{aligned}
$$

where $x^{*}$ is a fixed $\Delta_{1}^{1}$ point in $X$. It follows that $O$ is a $\Delta_{1}^{1}\left(x_{0}\right)$, open set such that $E \subseteq O \subseteq\{\tau<\infty\}$. Hence $\bar{M}(E)\left(x_{0}\right) \leq M(O)\left(x_{0}\right)$, so there is $\nu \in \Sigma\left(x_{0}\right)$ such that

$$
\nu(O) \geq \bar{M}(E)\left(x_{0}\right)-\varepsilon / 3 .
$$

Now

$$
\nu(O)=\int_{\{\tau<\infty\}} \nu\left[p_{\tau}\right]\left(O p_{\tau}\right)\left(h_{\tau}\right) d \nu(h) .
$$

If $\tau(h)<\infty$ and $\nu\left[p_{\tau}\right] \in \Sigma\left(h_{\tau}\right)$, then it follows from the definition of $O$ that

$$
\nu\left[p_{\tau}\right]\left(O p_{\tau}\right) \leq g\left(\left(h_{1}, h_{2}, \ldots, h_{\tau}, x^{*}, x^{*}, \ldots\right)\right)+\varepsilon / 3=g(h)+\varepsilon / 3 .
$$

Consequently, since $\Sigma$ is a global gambling house, we have

$$
\nu\left(\left\{h \in H: \tau(h)<\infty \& \nu\left[p_{\tau}\right]\left(O p_{\tau}\right) \leq g(h)+\varepsilon / 3\right\}\right)=\nu(\{\tau<\infty\}) .
$$

Hence,

$$
\begin{aligned}
\bar{M}(E)\left(x_{0}\right)-\frac{\varepsilon}{3} & \leq \int_{\{\tau<\infty\}} g(h) d \nu(h)+\frac{\varepsilon}{3} \leq \sup _{\mu \in \Sigma\left(x_{0}\right)} \int_{\{\tau<\infty\}} g(h) d \mu(h)+\frac{\varepsilon}{3} \\
& \leq \sup _{\mu \in \Sigma\left(x_{0}\right)} \int_{\{\tau<\infty\}} \bar{M}\left(E p_{\tau}\right)\left(h_{\tau}\right) d \mu(h)+\frac{2 \varepsilon}{3},
\end{aligned}
$$

where the last inequality involves an application of Lemma 4.3. It follows that

$$
\bar{M}(E)\left(x_{0}\right) \leq \sup _{\mu \in \Sigma\left(x_{0}\right)} \int_{\{\tau<\infty\}} \bar{M}\left(E p_{\tau}\right)\left(h_{\tau}\right) d \mu(h)+\varepsilon .
$$

Since $\varepsilon$ is an arbitrary positive rational, this completes the proof.

Theorem 4.4 is a significant generalization of Theorem 4.6 of [12].

Corollary 4.5. If $E \subseteq H$ is $\Sigma_{1}^{1}$ and $\tau$ is a $\Delta_{1}^{1}$-recursive stop rule, then

$$
\bar{M}(E)(x) \leq \sup _{\mu \in \Sigma(x)} \int \bar{M}\left(E p_{\tau}\right)\left(h_{\tau}\right) d \mu(h)
$$

for every $x \in X$.

\section{EFFECTIVE $G_{\delta}$ 's}

This section will be devoted to the proof of the effective refinement of Theorem 1.1 in the special case when $A$ is a $\Delta_{1}^{1}$-interesection of $\Delta_{1}^{1}$, open subsets of $H$. 
Theorem 5.1. Let $X=2^{\omega}, H=2^{\omega} \times 2^{\omega} \times \cdots$, and $\Sigma \subseteq X \times P(H)$ a $\Sigma_{1}^{1}$, global gambling house. Let $V_{n}$ be open subsets of $H$ such that $V_{n} \supseteq V_{n+1}, n \in N$, and assume that the relation $h \in V_{n}$ is $\Delta_{1}^{1}$ in $N \times H$. Set $G=\bigcap_{n \in N} V_{n}$. Then $\bar{M}(G)=M(G)$.

Proof. By Corollary 3.2, for each $n \in N$, there is a $\Delta_{1}^{1}$-recursive stopping time $\tau_{n}$ such that $V_{n}=\left\{\tau_{n}<\infty\right\}$. We may assume without loss of generality that $\tau_{n}<\tau_{n+1}$ on $V_{n+1}$ for all $n \in N$.

Fix $x_{0} \in X$ and $\varepsilon>0$. It will suffice to prove that $M(G)\left(x_{0}\right) \geq \bar{M}(G)\left(x_{0}\right)-$ $\varepsilon$. We will do this by defining $\mu \in \Sigma\left(x_{0}\right)$ such that

$$
\mu(G) \geq \bar{M}(G)\left(x_{0}\right)-\varepsilon .
$$

The definition of $\mu$ will involve repeated applications of Theorem 4.4.

To start with, use Theorem 4.4 to choose $\lambda \in \Sigma\left(x_{0}\right)$ such that

$$
\int_{\left\{\tau_{1}<\infty\right\}} \bar{M}\left(G p_{\tau_{1}}\right)\left(h_{\tau_{1}}\right) d \lambda(h)>\bar{M}(G)\left(x_{0}\right)-\varepsilon / 2 .
$$

Next, define $f: H \times N \times H^{\prime} \rightarrow[0,1]$ as follows:

$$
f\left(h, m, h^{\prime}\right)=\left\{\begin{array}{rr}
\bar{M}\left(G h_{1} h_{2} \cdots h_{m} p_{\tau_{n+1}\left[h_{1}, h_{2}, \ldots, h_{m}\right]}\left(h^{\prime}\right)\right)\left(h_{\tau_{n+1}\left[h_{1}, h_{2}, \ldots, h_{m}\right]}^{\prime}\right) \\
0 & \text { if } m<\tau_{n+1}\left(\left(h_{1} h_{2} \cdots h_{m}\right) h^{\prime}\right)<\infty \\
0 & \text { otherwise. }
\end{array}\right.
$$

Using Lemma 4.1, one verifies that $f$ is an upper analytic function. It then follows from a selection theorem [12, Lemma 2.1] (see also [1, Proposition 7.50]) that there is an analytically measurable function $\psi: H \times N \rightarrow P(H)$ such that $\psi(h, m) \in \Sigma\left(h_{m}\right)$ and

$$
\int f\left(h, m, h^{\prime}\right) \psi(h, m)\left(d h^{\prime}\right)>\sup _{\nu \in \Sigma\left(h_{m}\right)} \int f\left(h, m, h^{\prime}\right) \nu\left(d h^{\prime}\right)-\frac{\varepsilon}{2^{n+1}}
$$

for every $h \in H$ and $m \in N$. The function $\psi$ may not be adapted, but this is easily set right by defining $\phi_{n}: H \times N \rightarrow P(H)$ as follows:

$$
\phi_{n}(h, m)=\psi\left(\left(h_{1}, h_{2}, \ldots, h_{m}, x^{*}, x^{*}, \ldots\right), m\right),
$$

where $x^{*}$ is an arbitrary fixed point in $X$. We then have

$$
\int f\left(h, m, h^{\prime}\right) \phi_{n}(h, m)\left(d h^{\prime}\right)>\sup _{\nu \in \Sigma\left(h_{m}\right)} \int f\left(h, m, h^{\prime}\right) \nu\left(d h^{\prime}\right)-\frac{\varepsilon}{2^{n+1}}
$$

for all $h \in H$ and $m \in N$.

It now follows from (5.2), (5.3), and (5.4) that, if $\tau_{n+1}(h)>m$, then

$$
\begin{array}{r}
\int_{\left\{\tau_{n+1}\left[h_{1}, h_{2}, \ldots, h_{m}\right]<\infty\right\}} \bar{M}\left(G h_{1} h_{2} \cdots h_{m} p_{\tau_{n+1}\left[h_{1}, h_{2}, \ldots, h_{m}\right]}\left(h^{\prime}\right)\right) \\
\left(h_{\tau_{n+1}\left[h_{1}, h_{2}, \ldots, h_{m}\right]}^{\prime}\right) \phi_{n}(h, m)\left(d h^{\prime}\right) \\
>\sup _{\nu \in \Sigma\left(h_{m}\right)} \int_{\left\{\tau_{n+1}\left[h_{1}, h_{2}, \ldots, h_{m}\right]<\infty\right\}} \bar{M}\left(G h_{1} h_{2} \cdots h_{m} p_{\tau_{n+1}\left[h_{1}, h_{2}, \ldots, h_{m}\right]}\left(h^{\prime}\right)\right) \\
\geq \bar{M}\left(G h_{1} h_{2} \cdots h_{m}\right)\left(h_{m}\right)-\varepsilon / 2^{n+1}
\end{array}
$$


where the last inequality is obtained by an application of a relativized version of Theorem 4.4 to the $\Delta_{1}^{1}\left(\left\langle h_{1}, h_{2}, \ldots, h_{m}\right\rangle\right)$ set $G h_{1} h_{2} \cdots h_{m}$ and the $\Delta_{1}^{1}\left(\left\langle h_{1}, h_{2}, \ldots, h_{m}\right\rangle\right)$-recursive stopping time $\tau_{n+1}\left[h_{1}, h_{2}, \ldots, h_{m}\right]$. Hence, for any $h$ such that $\tau_{n}(h)<\infty$, we can set $m=\tau_{n}(h)$ in (5.5) to obtain

$$
\begin{gathered}
\int_{\left\{\tau_{n+1}\left[p_{\tau_{n}}(h)\right]<\infty\right\}} \bar{M}\left(G p_{\tau_{n}}(h) p_{\tau_{n+1}\left[p_{\tau_{n}}(h)\right]}\left(h^{\prime}\right)\right) \\
\left(h_{\tau_{n+1}\left[p_{\tau_{n}}(h)\right]}^{\prime}\right) \phi_{n}\left(h, \tau_{n}(h)\right)\left(d h^{\prime}\right) \\
>\bar{M}\left(G p_{\tau_{n}}(h)\right)\left(h_{\tau_{n}}\right)-\varepsilon / 2^{n+1} .
\end{gathered}
$$

Let $\mu$ be the sequential composition of $\left(\lambda, \phi_{n}, \tau_{n}\right)$. Plainly, $\mu \in \Sigma\left(x_{0}\right)$, since $\Sigma$ is a global gambling house. We will now verify (5.1). In the calculations to follow, expectations and conditional expectations will be with respect to the probability measure $\mu$ on $H$.

Define random variables $Y_{n}$ on $H, n \geq 1$, as follows:

$$
Y_{n}(h)= \begin{cases}\bar{M}\left(G p_{\tau_{n}}(h)\right)\left(h_{\tau_{n}}\right) & \text { if } h \in V_{n}, \\ 0 & \text { otherwise }\end{cases}
$$

Then, a restatement of $(5.2)$ gives us

$$
E\left(Y_{1}\right)>\bar{M}(G)\left(x_{0}\right)-\varepsilon / 2,
$$

while (5.6) yields for almost all $(\mu) h \in V_{n}$,

$$
E\left(Y_{n+1} \mid p_{\tau_{n}}\right)>\bar{M}\left(G p_{\tau_{n}}(h)\right)\left(h_{\tau_{n}}\right)-\varepsilon / 2^{n+1},
$$

where the conditional expectation above is with respect to the pre- $\tau_{n} \sigma$-field. Hence

so that

$$
E\left(Y_{n+1} \mid p_{\tau_{n}}\right)>Y_{n}-\varepsilon / 2^{n+1} \quad \text { a.s. }(\mu)
$$

$$
E\left(Y_{n+1}\right)>E\left(Y_{n}\right)-\varepsilon / 2^{n+1} .
$$

It then follows from (5.7) and (5.8) that

$$
E\left(Y_{n+1}\right)>E\left(Y_{1}\right)-\left(\varepsilon / 2^{2}+\varepsilon / 2^{3}+\cdots+\varepsilon / 2^{n+1}\right)>\bar{M}(G)\left(x_{0}\right)-\varepsilon
$$

for every $n \geq 0$. Hence

$$
\limsup _{n} E\left(Y_{n}\right) \geq \bar{M}(G)\left(x_{0}\right)-\varepsilon .
$$

Now, for each $n \in N,\left\{Y_{n}>0\right\} \subseteq V_{n}$. Since $0 \leq Y_{n} \leq 1$, it follows that $\mu\left(V_{n}\right) \geq E\left(Y_{n}\right)$ for every $n \in N$. Hence,

$$
\mu(G)=\lim _{n} \mu\left(V_{n}\right) \geq \limsup _{n} E\left(Y_{n}\right) \geq \bar{M}(G)\left(x_{0}\right)-\varepsilon,
$$

where the equality uses the fact that $G$ is the decreasing intersection of the sets $V_{n}$ and the last equation is by virtue of (5.9). So (5.1) is verified and the proof is complete.

\section{EFFECTIVE $G_{\delta \sigma}$ 's AND THE "GOING UP" PROPERTY}

This section contains the second step in our program of proving the effective version of Theorem 1.1. We start with a definition. We remind the reader that $X, H$, and $\Gamma$ are as in the previous section.

A sequence $\left(E_{n}\right)_{n \in N}$ of subsets of $H$ is a $\Sigma_{1}^{1}\left(\Delta_{1}^{1}\right)$ sequence if the relation $h \in E_{n}$ is $\Sigma_{1}^{1}\left(\Delta_{1}^{1}\right)$ in $N \times H$. 
Lemma 6.1. If $\left(E_{n}\right)$ is a $\Sigma_{1}^{1}$ sequence of subsets of $H$ and $\bar{M}\left(E_{n}\right)(x)=0$ for each $n \in N$, then $\bar{M}\left(\bigcup_{n \in N} E_{n}\right)(x)=0$.

Proof. Let $\varepsilon>0$ be rational. Define $P \subseteq N \times \omega$ by

$$
(n, m) \in P \leftrightarrow(x, m) \in \bar{W} \& E_{n} \subseteq \bar{C}_{x, m} \&(\forall \mu \in \Sigma(x))\left(\mu\left(\bar{C}_{x, m}\right)<\frac{\varepsilon}{2^{n}}\right) .
$$

Then $P$ is $\Pi_{1}^{1}(x)$, and the hypothesis implies that $(\forall n \in N)(\exists m)[(n, m) \in P]$. So, by Kreisel's selection theorem, there is a $\Delta_{1}^{1}(x)$-recursive function $f: N \rightarrow$ $\omega$ such that $(\forall n \in N)[(n, f(n)) \in P]$. Let

$$
h \in O \leftrightarrow(\exists n \in N)\left[h \in \bar{C}_{x, f(n)}\right] .
$$

Then $O$ is a $\Delta_{1}^{1}(x)$, open set containing $\bigcup_{n \in N} E_{n}$. Hence

$$
\bar{M}\left(\bigcup_{n \in N} E_{n}\right)(x) \leq M(O)(x) \leq \sum_{n} M\left(\bar{C}_{x, f(n)}\right)(x) \leq \varepsilon .
$$

Since $\varepsilon$ is arbitrary, this completes the proof of the lemma.

Lemma 6.2. Suppose that the relation $h \in B_{n, m}$ is $\Delta_{1}^{1}$ in $H \times N \times N$ and that $B_{n, m}$ is open in $H$ for every $n, m \in N$. Let $E_{n}=\bigcap_{m \in N} B_{n, m}, n \in N$. If $M\left(\bigcup_{n \in N} E_{n}\right)(x)=0$, then $\bar{M}\left(\bigcup_{n \in N} E_{n}\right)(x)=0$.

Proof. By Theorem 5.1, $\bar{M}\left(E_{n}\right)(x)=M\left(E_{n}\right)(x)=0$ for every $n \in N$. The assertion of the lemma now follows from Lemma 6.1.

Let $E \subseteq H$ be $\Sigma_{1}^{1}$. Fix $\varepsilon>0$ rational. Define a stopping time $\tau_{\varepsilon}$ as follows:

$$
\tau_{\varepsilon}(h)=\left\{\begin{array}{c}
\text { least } k \in N \text { such that } M\left(E h_{1} h_{2} \cdots h_{k}\right)\left(h_{k}\right)>1-\varepsilon, \\
\infty \text { otherwise. }
\end{array}\right.
$$

Lemma 6.3. (a) The set $\left\{(h, n) \in H \times N: \tau_{\varepsilon}(h) \leq n\right\}$ is $\Sigma_{1}^{1}$.

(b) The set $\left\{\tau_{\varepsilon}<\infty\right\}$ is $\Sigma_{1}^{1}$ in $H$.

Proof. The relation $M\left(E h_{1} h_{2} \cdots h_{k}\right)\left(h_{k}\right)>1-\varepsilon$ in $h, k$ can be rewritten as

$$
\left(\exists \mu \in \Sigma\left(h_{k}\right)\right)\left(\mu\left(E h_{1} h_{2} \cdots h_{k}\right)>1-\varepsilon\right) .
$$

This is clearly $\Sigma_{1}^{1}$ in $H \times N$. The assertion in (a) follows immediately from this and (b) follows from (a).

Lemma 6.4. Let $E$ and $\tau_{\varepsilon}$ be as above. Then $M\left(E \cap\left\{\tau_{\varepsilon}=\infty\right\}\right)(x)=0$ for every $x \in X$.

Proof. Towards a contradiction, assume there is an $x$ such that

$$
M\left(E \cap\left\{\tau_{\varepsilon}=\infty\right\}\right)(x)>0 .
$$

So there exists $\mu \in \Sigma(x)$ such that $\mu\left(E \cap\left\{\tau_{\varepsilon}=\infty\right\}\right)>0$. By the Levy zero-one law, as $n \rightarrow \infty$,

$$
\mu\left[p_{n}(h)\right]\left[\left(E \cap\left\{\tau_{\varepsilon}=\infty\right\}\right) p_{n}(h)\right] \rightarrow\left(E \cap\left\{\tau_{\varepsilon}=\infty\right\}\right)(h) \quad \text { a.s. }(\mu),
$$

where the indicator function of a set $C$ is being donated by the same symbol. Denote by $C_{1}$ the subset of $H$ where convergence holds in (6.1), so that $\mu\left(C_{1}\right)=1$. Let $C_{2}$ be the set of $h \in H$ such that $\mu\left[p_{n}(h)\right] \in \Sigma\left(h_{n}\right)$ for every 
$n \in N$. Since $\Sigma$ is a global gambling house, it follows that $\mu\left(C_{2}\right)=1$. Consequently, $\mu\left(C_{1} \cap C_{2} \cap E \cap\left\{\tau_{\varepsilon}=\infty\right\}\right)>0$. Pick $h^{*}$ in $C_{1} \cap C_{2} \cap E \cap\left\{\tau_{\varepsilon}=\infty\right\}$. So, by (6.1), there is $m \geq 1$ such that

$$
\mu\left[p_{m}\left(h^{*}\right)\right]\left[\left(E \cap\left\{\tau_{\varepsilon}=\infty\right\}\right) p_{m}\left(h^{*}\right)\right]>1-\varepsilon .
$$

This implies that $\mu\left[p_{m}\left(h^{*}\right)\right]\left(E p_{m}\left(h^{*}\right)\right)>1-\varepsilon$, so $M\left(E p_{m}\left(h^{*}\right)\right)\left(h_{m}^{*}\right)>1-\varepsilon$, since $\mu\left[p_{m}\left(h^{*}\right)\right] \in \Sigma\left(h_{m}^{*}\right)$. But then $\tau_{\varepsilon}\left(h^{*}\right) \leq m$, contradicting the fact that $\tau_{\varepsilon}\left(h^{*}\right)=\infty$. This completes the proof.

Lemma 6.5. $M\left(\left\{\tau_{\varepsilon}<\infty\right\}\right)(x) \leq M\left(E \cap\left\{\tau_{\varepsilon}<\infty\right\}\right)(x)+2 \varepsilon$ for every $x \in X$.

Proof. We write $\tau$ for $\tau_{\varepsilon}$. Choose $\lambda \in \Sigma(x)$ such that

$$
\lambda(\{\tau<\infty\})>M(\{\tau<\infty\})(x)-\varepsilon / 2 .
$$

Next, use a selection theorem [12, Lemma 2.1 or 1, Proposition 7.50] to find, for each $n \in N$, an analytically measurable selector $\psi_{n}: H \rightarrow P(H)$ for the set $\left\{(h, \mu) \in H \times P(H): \mu \in \Sigma\left(h_{n}\right)\right\}$ such that

$$
\psi_{n}(h)\left(E p_{n}(h)\right) \geq M\left(E p_{n}(h)\right)\left(h_{n}\right)-\varepsilon / 2
$$

for every $h \in H$.

Let $x^{*}$ be a fixed element of $X$. By the von-neumann selection theorem [15, 4E.9], fix an analytically measurable selector $f^{*}: H \rightarrow P(H)$ for the set $\Sigma$. Define

$$
\phi_{1}(h, k)=\psi_{k}\left(\left(h_{1}, h_{2}, \ldots, h_{k}, x^{*}, x^{*}, \ldots\right)\right)
$$

and, for $n \geq 2$,

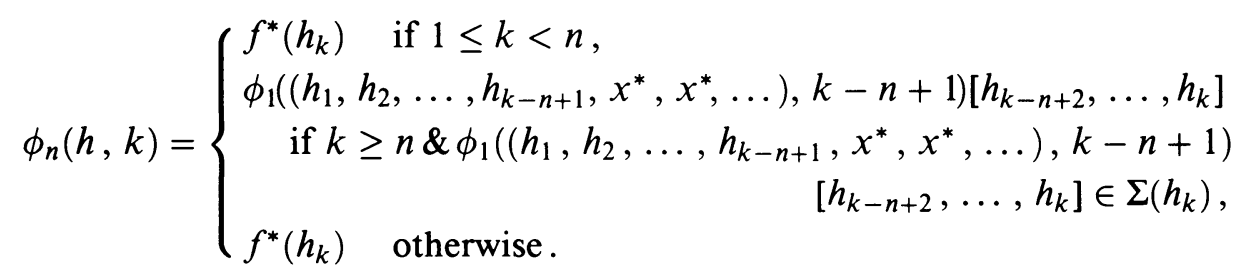

Set $\tau_{1}=\tau$, and, for $n \geq 2, \tau_{n}=\tau_{1}+n-1$. Then it is straightforward to verify that $\lambda, \phi_{n}$, and $\tau_{n}$ satisfy the assumptions of $(\mathrm{b})$ in the definition of a global gambling house. Let $\mu$ be the sequential composition of $\left(\lambda, \phi_{n}, \tau_{n}\right)$, so $\mu \in \Sigma(x)$.

Hence,

$$
\begin{aligned}
M(E \cap\{\tau<\infty\})(x) & \geq \mu(E \cap\{\tau<\infty\})=\int_{\{\tau<\infty\}} \mu\left[p_{\tau}\right]\left(E p_{\tau}\right) d \lambda \\
& \geq(1-3 \varepsilon / 2) \lambda(\{\tau<\infty\}) \geq M(\{\tau<\infty\})(x)-2 \varepsilon .
\end{aligned}
$$

This completes the proof.

We now give the main result of this section.

Theorem 6.6. Let $X=2^{\omega}, H=2^{\omega} \times 2^{\omega} \times \cdots$, and $\Sigma \subseteq X \times P(H)$ be a $\Sigma_{1}^{1}$, global gambling house. Suppose that the relation $h \in B_{n, m}$ is $\Delta_{1}^{1}$ in $H \times N \times N$ and that $B_{n, m}$ is open in $H$ for every $n, m \in N$. Let $E_{n}=\bigcap_{m \in N} B_{n, m}$, $n \in N$, and $E=\bigcup_{n \in N} E_{n}$. Then $\bar{M}(E)=M(E)$.

Proof. Fix $x_{0} \in X$ and $\varepsilon>0$ rational. Consider the stopping time $\tau_{\varepsilon}$ associated with the set $E$. By Lemma $6.3,\left\{\tau_{\varepsilon}<\infty\right\}$ is a $\Sigma_{1}^{1}$ set. So, by a 
relativized version of Lemma 4.3 in [12], there is a $\Delta_{1}^{1}\left(x_{0}\right)$ set $D$ in $H$ such that $\left\{\tau_{\varepsilon}<\infty\right\} \subseteq D$ and

$$
M(D)\left(x_{0}\right)<M\left(\left\{\tau_{\varepsilon}<\infty\right\}\right)\left(x_{0}\right)+\varepsilon .
$$

Now the $\Sigma_{1}^{1}$ set $\left\{\tau_{\varepsilon}<\infty\right\}$ can be separated from the $\Delta_{1}^{1}\left(x_{0}\right)$ set $D^{c}$ by an open set. Hence, by a relativized version of Lemma 3.1 , there is a $\Delta_{1}^{1}\left(x_{0}\right)$-recursive stopping time $t$ such that $\left\{\tau_{\varepsilon}<\infty\right\} \subseteq\{t<\infty\} \subseteq D$. Set $O=\{t<\infty\}$. Then

$$
\begin{aligned}
M(O)\left(x_{0}\right) & <M\left(\left\{\tau_{\varepsilon}<\infty\right\}\right)\left(x_{0}\right)+\varepsilon \\
& \leq M\left(E \cap\left\{\tau_{\varepsilon}<\infty\right\}\right)\left(x_{0}\right)+3 \varepsilon \leq M(E \cap O)\left(x_{0}\right)+3 \varepsilon,
\end{aligned}
$$

where the second inequality is by virtue of Lemma 6.5. On the other hand,

$$
E \cap O^{c}=\left[\bigcup_{n \in N} \bigcap_{m \in N} B_{n, m}\right] \cap \bigcap_{k \in N}[\{t \geq k\}]=\bigcup_{n \in N} \bigcap_{m \in N} \bigcap_{k \in N}\left[B_{n, m} \cap\{t \geq k\}\right] .
$$

Moreover, by virtue of Lemma 6.4,

$$
M\left(E \cap O^{c}\right)\left(x_{0}\right) \leq M\left(E \cap\left\{\tau_{\varepsilon}=\infty\right\}\right)\left(x_{0}\right)=0 .
$$

This fact together with the representation of $E \cap O^{c}$ above enables us to invoke a relativized version of Lemma 6.2 and conclude that

$$
\bar{M}^{x_{0}}\left(E \cap O^{c}\right)\left(x_{0}\right)=0 .
$$

Now use (6.3) to obtain a $\Delta_{1}^{1}\left(x_{0}\right)$, open set $O^{\prime}$ containing $E \cap O^{c}$ and such that

$$
M\left(O^{\prime}\right)\left(x_{0}\right)<\varepsilon .
$$

Then $O \cup O^{\prime}$ is a $\Delta_{1}^{1}\left(x_{0}\right)$, open set and contains $E$. Hence,

$$
\begin{aligned}
\bar{M}(E)\left(x_{0}\right) & \leq M\left(O \cup O^{\prime}\right)\left(x_{0}\right) \leq M(O)\left(x_{0}\right)+M\left(O^{\prime}\right)\left(x_{0}\right) \\
& \leq M(E \cap O)\left(x_{0}\right)+4 \varepsilon \leq M(E)\left(x_{0}\right)+4 \varepsilon,
\end{aligned}
$$

where the third inequality is by virtue of $(6.2)$ and (6.4). Since $\varepsilon$ is an arbitrary rational, this proves that $\bar{M}(E)\left(x_{0}\right) \leq M(E)\left(x_{0}\right)$. As the inequality in the opposite direction is obvious, tilis completes the proof.

The next result is the raison d'être for this intermediate step in the proof of the effective refinement of Theorem 1.1.

Corollary 6.7. Let $\left(A_{n}\right)$ be a $\Sigma_{1}^{1}$ sequence of subsets of $H$ such that $A_{n} \subseteq A_{n+1}$, $n \in N$. Let $A=\bigcup_{n \in N} A_{n}$. Then $\bar{M}(A)=\lim \bar{M}\left(A_{n}\right)$.

Proof. Fix $x_{0} \in X$. Plainly, $\bar{M}(A)\left(x_{0}\right) \geq \lim \bar{M}\left(A_{n}\right)\left(x_{0}\right)$. It remains only to prove the inequality in the opposite direction. Let $r$ be a rational in $[0,1]$ such that $\bar{M}(A)\left(x_{0}\right)>r$. We need to show that $\lim \bar{M}\left(A_{n}\right)\left(x_{0}\right) \geq r$. Suppose not. Then, for each $n \in N, \bar{M}\left(A_{n}\right)\left(x_{0}\right)<r$. Define $P \subseteq N \times \omega$ as follows:

$$
(n, m) \in P \leftrightarrow\left(x_{0}, m\right) \in \bar{W} \& A_{n} \subseteq \bar{C}_{x_{0}, m} \&\left(\forall \mu \in \Sigma\left(x_{0}\right)\right)\left(\mu\left(\bar{C}_{x_{0}, m}\right)<r\right) .
$$

Plainly, $P$ is $\Pi_{1}^{1}\left(x_{0}\right)$ and $(\forall n \in N)(\exists m)[(n, m) \in P]$. So, by Kreisel's selection theorem, there is a $\Delta_{1}^{1}\left(x_{0}\right)$-recursive function $f: N \rightarrow \omega$ such that $(\forall n \in$ $N)[(n, f(n)) \in P]$. Let

$$
h \in B \leftrightarrow(\exists k \in N)(\forall n \geq k)\left[h \in \bar{C}_{x_{0}, f(n)} .\right.
$$


Then $A \subseteq B$, and by Theorem 6.6 (relativized to $\left.x_{0}\right), \bar{M}^{x_{0}}(B)\left(x_{0}\right)=M(B)\left(x_{0}\right)$. Hence,

$$
\begin{aligned}
\bar{M}(A)\left(x_{0}\right) & \leq \bar{M}^{x_{0}}(B)\left(x_{0}\right)=M(B)\left(x_{0}\right)=\sup _{k} M\left(\bigcap_{n \geq k} \bar{C}_{x_{0}, f(n)}\right)\left(x_{0}\right) \\
& \leq \sup _{k} M\left(\bar{C}_{x_{0}, f(k)}\right)\left(x_{0}\right) \leq r
\end{aligned}
$$

which yields the desired contradiction.

\section{AN EFFECTIVE CAPACITABILITY THEOREM}

For fixed $x \in X$, the monotonicity property and the property stated in Corollary 6.7 suggest that the set function $\bar{M}(\cdot)(x)$ may be an "effective" capacity in the sense of Louveau [9]. However, this is not so, as the "going down" property along decreasing sequences of $T_{1}$-compacts fails to hold in general (see [13]). In consequence, the "effective" capacitability theorem of Louveau cannot be applied directly, as Choquet's capacitability theorem was in some special cases (see [13, Theorem 3.6]), to prove Theorem 1.1. Nonetheless, we will now combine the proof of the abstract capacitability theorem -or, to more precisely identify the provenance of these ideas, Sierpinski's proof that an analytic set is measurable [21, pp. 48-50]-with the proof of Theorem 5.1 to produce a proof of the effective refinement of Theorem 1.1. But first we must explain some more notation from the effective theory.

Let $U: \omega^{\omega} \times H \rightarrow \omega$ be a $\Pi_{1}^{1}$-recursive partial function such that whenever $\alpha \in \omega^{\omega}$ and $f: H \rightarrow \omega$ is a $\Pi_{1}^{1}(\alpha)$-recursive partial function, there is a $\Delta_{1}^{1}(\alpha)$ point $\beta \in \omega^{\omega}$ such that if $f(h)$ is defined, then $U(\beta, h)$ is defined and $f(h)=$ $U(\beta, h)$. Following Kleene, we shall denote the partial function $U(\beta, \cdot)$ by $\{\beta\}$. We write $\{\beta\}(h) \downarrow$ to mean that $\{\beta\}$ is defined at $h$. Next, fix a $\Pi_{1}^{1}$ recursive partial function $d: \omega^{\omega} \times \omega \rightarrow \omega^{\omega}$ such that $d(\alpha, \cdot)$ enumerates the $\Delta_{1}^{1}(\alpha)$ points of $\omega^{\omega}$. The existence of $U$ is established in [15, 7A.1] and that of $d$ is proved in [15, 4D.2].

Here at last is the effective refinement of Theorem 1.1 .

Theorem 7.1. Let $X=2^{\omega}, H=2^{\omega} \times 2^{\omega} \times \cdots$, and $\Sigma \subseteq X \times P(H)$ be a $\Sigma_{1}^{1}$, global gambling house. If $A \subseteq H$ is a $\Sigma_{1}^{1}$ set, then $\bar{M}(A)=M(A)$.

Proof. We use [15, 4A.1] to write $A$ as follows:

$$
h \in A \leftrightarrow\left(\exists \alpha \in \omega^{N}\right)(\forall n \in N)[h \in B(\bar{\alpha}(n))],
$$

where $h \in B(s)$ is a $\Delta_{1}^{1}$ relation in $\bigcup_{n \in \omega} \omega^{n} \times H, B(s)$ is clopen in $H$ for each fixed $s \in \bigcup_{n \in \omega} \omega^{n}$, and $s \supseteq t$ implies $B(s) \subseteq B(t)$. Define

$$
\begin{aligned}
& h \in A(s) \leftrightarrow(\exists m)\left[|s|=m \&\left(\exists i_{1} \leq s_{1}\right)\left(\exists i_{2} \leq s_{2}\right) \cdots\left(\exists i_{m} \leq s_{m}\right)(\exists \alpha)\right. \\
& \left.(\forall n \in N)\left(h \in B\left(\left(i_{1}, i_{2}, \ldots, i_{m}\right) \bar{\alpha}(n)\right)\right)\right] .
\end{aligned}
$$

Plainly, the relation $h \in A(s)$ is $\Sigma_{1}^{1}$ in $\bigcup_{n \in \omega} \omega^{n} \times H$.

Fix $x_{0} \in X$ and $\varepsilon>0$. We will construct $\mu \in \Sigma\left(x_{0}\right)$ such that

$$
\mu(A) \geq \bar{M}(A)\left(x_{0}\right)-\varepsilon .
$$

The definition of $\mu$ depends on an inductive construction of various objects. The conditions that these objects have to satisfy are stated in the next lemma. 
Lemma 7.2. Let $x_{0} \in X, \varepsilon>0, \lambda \in \Sigma\left(x_{0}\right), m_{1}^{*} \in \omega, m_{n+1}^{*}: H \rightarrow \omega, K_{n} a$ clopen subset of $H, \tau_{n}$ a stop rule on $H$, and $\phi_{n}: H \times N \rightarrow P(H)$ such that

(a) for every $n \in N, m_{n+1}^{*}, K_{n}, \tau_{n}$, and $\phi_{n}$ are universally measurable, $K_{n+1} \subseteq K_{n}, \tau_{n}<\tau_{n+1}, K_{n}$, and $m_{n+1}^{*}$ are determined by time $\tau_{n}, \phi_{n}(h, k) \in$ $\Sigma\left(h_{k}\right)$ for every $k \in N$, and $\phi_{n}$ is adapted;

(b) $h \in K_{n}$ iff $h \in B\left(\left(i_{1}, i_{2}, \ldots, i_{n}\right)\right)$ for some $i_{1} \leq m_{1}^{*}, i_{2} \leq m_{2}^{*}(h), \ldots$, $i_{n} \leq m_{n}^{*}(h)$

(c) $\int_{K_{1}} \bar{M}\left(A\left(\left(m_{1}^{*}\right)\right) q_{1}(h)\right)\left(h_{\tau_{1}}\right) d \lambda(h)>\bar{M}(A)\left(x_{0}\right)-\varepsilon / 2$; and

(d) for every $n \in N$ and $h \in H$,

$$
\begin{gathered}
\int_{K_{n+1} q_{n}(h)} \bar{M}\left(A\left(\left(m_{1}^{*}, m_{2}^{*}(h), \ldots, m_{n+1}^{*}(h)\right)\right) q_{n}(h) p_{\tau_{n+1}\left[q_{n}(h)\right]}\right) \\
\left(h_{\tau_{n+1}\left[q_{n}(h)\right]}^{\prime}\right) \phi_{n}\left(h, \tau_{n}(h)\right)\left(d h^{\prime}\right) \\
>\bar{M}\left(A\left(\left(m_{1}^{*}, m_{2}^{*}(h), \ldots, m_{n}^{*}(h)\right)\right) q_{n}(h)\right)\left(h_{\tau_{n}}\right)-\frac{\varepsilon}{2^{n+1}},
\end{gathered}
$$

where $q_{n}(h)=p_{\tau_{n}}(h), n \in N$.

Let $\mu$ be the sequential composition of $\left(\lambda, \phi_{n}, \tau_{n}\right)$. Then

(i) $\bigcap_{n \in N} K_{n} \subseteq A$, and

(ii) $\mu\left(\bigcap_{n \in N} K_{n}\right) \geq \bar{M}(A)\left(x_{0}\right)-\varepsilon$.

Proof. To prove (i), let $h \in \bigcap_{n \in N} K_{n}$ and define

$$
\begin{array}{r}
S=\left\{s \in \bigcup_{n \in \omega} \omega^{n}:(\exists k)\left[|s|=k \& s_{1} \leq m_{1}^{*} \& s_{2} \leq m_{2}^{*}(h)\right.\right. \\
\left.\left.\& \cdots \& s_{k} \leq m_{k}^{*}(h) \& h \in B(s)\right]\right\} .
\end{array}
$$

Then $S$ is a finitely splitting tree and contains finite sequences of every length. So, by König's Lemma [15, 4F.9], there exists $\alpha \in \omega^{N}$ such that $\bar{\alpha}(n) \in S$ for every $n \in N$. Hence, $h \in B(\bar{\alpha}(n))$ for every $n \in N$, so that $h \in A$.

We will now prove (ii). Define random variables $Y_{n}$ on $H, n \geq 1$ as follows:

$$
Y_{n}(h)= \begin{cases}\bar{M}\left(A\left(m_{1}^{*}, m_{2}^{*}(h), \ldots, m_{n}^{*}(h)\right) q_{n}(h)\right)\left(h_{\tau_{n}}\right) & \text { if } h \in K_{n}, \\ 0 & \text { otherwise }\end{cases}
$$

In what follows, expectations and conditional expectations are with respect to the probability measure $\mu$ on $H$. According to (c), we have

$$
E\left(Y_{1}\right)>\bar{M}(A)\left(x_{0}\right)-\varepsilon / 2 \text {, }
$$

while (d) yields for almost all $(\mu) h \in H$,

$$
E\left(Y_{n+1} \mid p_{\tau_{n}}\right)>\bar{M}\left(A\left(m_{1}^{*}, m_{2}^{*}(h), \ldots, m_{n}^{*}(h)\right) q_{n}(h)\right)\left(h_{\tau_{n}}\right)-\frac{\varepsilon}{2^{n+1}},
$$

where the conditional expectation is with respect to the pre- $\tau_{n} \sigma$-field. Hence

$$
E\left(Y_{n+1} \mid p_{\tau_{n}}\right)>Y_{n}-\varepsilon / 2^{n+1}
$$

so that

$$
E\left(Y_{n+1}\right)>E\left(Y_{n}\right)-\varepsilon / 2^{n+1}
$$


Consequently,

$$
E\left(Y_{n+1}\right)>E\left(Y_{1}\right)-\left(\frac{\varepsilon}{2^{2}}+\frac{\varepsilon}{2^{3}}+\cdots+\frac{\varepsilon}{2^{n+1}}\right)
$$

for every $n \geq 0$, and so

$$
\limsup _{n} E\left(Y_{n}\right) \geq \bar{M}(A)\left(x_{0}\right)-\varepsilon .
$$

Now for each $n \in N,\left\{Y_{n}>0\right\} \subseteq K_{n}$. Since $0 \leq Y_{n} \leq 1$, it follows that $\mu\left(K_{n}\right) \geq E\left(Y_{n}\right)$ for every $n \in N$. Hence,

$$
\mu\left(\bigcap_{n \in N} K_{n}\right)=\lim _{n} \mu\left(K_{n}\right) \geq \limsup _{n} E\left(Y_{n}\right) \geq \bar{M}(A)\left(x_{0}\right)-\varepsilon,
$$

where the first inequality is by virtue of (7.4). This establishes (ii) and completes the proof of the lemma.

We return to the proof of Theorem 7.1. Since $\Sigma$ is a global gambling house, $\mu$, constructed in Lemma 7.2, belongs to $\Sigma\left(x_{0}\right)$. So (7.3) is an immediate consequence of Lemma 7.2. It therefore remains to show that the hypotheses of Lemma 7.2 are satisfied. We begin with a lemma.

Lemma 7.3. For every $n \geq 2$, there is a $\Delta_{1}^{1}$-recursive function $g_{n}: H \times \omega^{n} \times N \rightarrow$ $\omega^{\omega}$ such that if

$$
g_{n}\left(h, m_{1}, m_{2}, \ldots, m_{n}, k\right)=\alpha,
$$

then $\alpha$ is a $\Delta_{1}^{1}\left(\left\langle h_{1}, h_{2}, \ldots, h_{k}\right\rangle\right)$ point and $\{\alpha\}$ is a $\Delta_{1}^{1}\left(\left\langle h_{1}, h_{2}, \ldots, h_{k}\right\rangle\right)$ recursive stop rule $\tau$ on $H$ such that the $\Delta_{1}^{1}\left(\left\langle h_{1}, h_{2}, \ldots, h_{k}\right\rangle\right)$, clopen set

$$
\bigcup_{i_{1}=0}^{m_{1}} \bigcup_{i_{2}=0}^{m_{2}} \cdots \bigcup_{i_{n}=0}^{m_{n}} B\left(\left(i_{1}, i_{2}, \ldots, i_{n}\right)\right) h_{1} h_{2} \ldots h_{k}
$$

is determined by time $\tau$. Furthermore, if $h \equiv_{k} h^{\prime}$, then $g_{n}\left(h, m_{1}, m_{2}, \ldots, m_{n}, k\right)$ $=g_{n}\left(h^{\prime}, m_{1}, m_{2}, \ldots, m_{n}, k\right)$.

Proof. Define $P \subseteq H \times \omega^{n} \times N \times \omega$ as follows:

$$
\begin{aligned}
& \left(h, m_{1}, m_{2}, \ldots, m_{n}, k, j\right) \in P \\
& \leftrightarrow d\left(\left\langle h_{1}, h_{2}, \ldots, h_{k}\right\rangle, j\right) \downarrow \&\left(\forall h^{\prime}\right)\left\{\left\{d\left(\left\langle h_{1}, h_{2}, \ldots, h_{k}\right\rangle, j\right)\right\}\left(h^{\prime}\right) \downarrow\right. \\
& \left.\&\left\{d\left(\left\langle h_{1}, h_{2}, \ldots, h_{k}\right\rangle, j\right)\right\}\left(h^{\prime}\right) \geq 1\right] \\
& \&\left(\forall h^{\prime}\right)\left(\forall h^{\prime \prime}\right)(\forall i)\left[\left\{d\left(\left\langle h_{1}, h_{2}, \ldots, h_{k}\right\rangle, j\right)\right\}\left(h^{\prime}\right)=i \& h^{\prime} \equiv{ }_{i} h^{\prime \prime}\right. \\
& \left.\rightarrow\left\{d\left(\left\langle h_{1}, h_{2}, \ldots, h_{k}\right\rangle, j\right)\right\}\left(h^{\prime \prime}\right)=i\right] \\
& \&\left(\forall h^{\prime}\right)(\forall i)\left[\left\{d\left(\left\langle h_{1}, h_{2}, \ldots, h_{k}\right\rangle, j\right)\right\}\left(h^{\prime}\right)=i\right. \\
& \rightarrow\left(\forall h^{\prime \prime}\right)\left(h^{\prime \prime} \equiv{ }_{i} h^{\prime} \rightarrow h^{\prime \prime} \in \bigcup_{i_{1}=0}^{m_{1}} \bigcup_{i_{2}=0}^{m_{2}} \cdots \bigcup_{i_{n}=0}^{m_{n}} B\left(\left(i_{1}, i_{2}, \ldots, i_{n}\right)\right) h_{1} h_{2} \cdots h_{k}\right) \\
& \left.\vee\left(\forall h^{\prime \prime}\right)\left(h^{\prime \prime} \equiv{ }_{i} h^{\prime} \rightarrow h^{\prime \prime} \notin \bigcup_{i_{1}=0}^{m_{1}} \bigcup_{i_{2}=0}^{m_{2}} \cdots \bigcup_{i_{n}=0}^{m_{n}} B\left(\left(i_{1}, i_{2}, \ldots, i_{n}\right)\right) h_{1} h_{2} \cdots h_{k}\right)\right] .
\end{aligned}
$$

Then $P$ is $\Pi_{1}^{1}$ and an application of a relativized version of Corollary 3.3 will show that

$$
(\forall h)\left(\forall m_{1}\right)\left(\forall m_{2}\right) \cdots\left(\forall m_{n}\right)(\forall k)(\exists j)\left[\left(h, m_{1}, m_{2}, \ldots, m_{n}, k, j\right) \in P\right] .
$$


So, by Kreisel's selection theorem, there is a $\Delta_{1}^{1}$-recursive function $f: H \times w^{n} \times$ $N \rightarrow w$ such that

$$
\begin{aligned}
& (\forall h)\left(\forall m_{1}\right)\left(\forall m_{2}\right) \cdots\left(\forall m_{n}\right)(\forall k) \\
& \quad\left[\left(h, m_{1}, m_{2}, \ldots, m_{n}, k, f\left(h, m_{1}, m_{2}, \ldots, m_{n}, k\right)\right) \in P\right] .
\end{aligned}
$$

Finally, set

$$
\begin{aligned}
& g_{n}\left(h, m_{1}, m_{2}, \ldots, m_{n}, k\right) L \\
& \quad=d\left(\left\langle h_{1}, h_{2}, \ldots, h_{k}\right\rangle, f\left(\bar{h}, m_{1}, m_{2}, \ldots, m_{n}, k\right)\right),
\end{aligned}
$$

where $\bar{h}=\left(h_{1}, h_{2}, \ldots, h_{k}, x^{*}, x^{*}, \ldots\right)$ and $x^{*}$ is a fixed $\Delta_{1}^{1}$ point in $X$. It is now easy to verify that $g_{n}$ satisfies the assertions of the lemma.

We are now ready to complete the proof of Theorem 7.1. Observe, first, that $(A(m))_{m \in \omega}$ is a $\Sigma_{1}^{1}$ sequence, $A(m) \subseteq A(m+1)$, and $\bigcup_{m \in \omega} A(m)=A$. Hence, by Corollary 6.7, there is $m_{1}^{*}$ such that

$$
\bar{M}\left(A\left(\left(m_{1}^{*}\right)\right)\right)\left(x_{0}\right)>\bar{M}(A)\left(x_{0}\right)-\varepsilon / 4 .
$$

Define $K_{1}$ as follows:

$$
h \in K_{1} \leftrightarrow\left(\exists i \leq m_{1}^{*}\right)[h \in B((i))],
$$

so $K_{1}$ is a $\Delta_{1}^{1}$, clopen set and $A\left(\left(m_{1}^{*}\right)\right) \subseteq K_{1}$. By Corollary 3.3, there is a $\Delta_{1}^{1}$ recursive stop rule $\tau_{1}$ such that $K_{1}$ is determined by time $\tau_{1}$. Apply Corollary 4.5 to the $\Sigma_{1}^{1}$ set $A\left(\left(m_{1}^{*}\right)\right)$ and $\Delta_{1}^{1}$-recursive stop rule $\tau_{1}$ at the point $x_{0}$ to get $\lambda \in \Sigma\left(x_{0}\right)$ such that

$$
\int \bar{M}\left(A\left(\left(m_{1}^{*}\right)\right) p_{\tau_{1}}\right)\left(h_{\tau_{1}}\right) d \lambda(h)>\bar{M}\left(A\left(\left(m_{1}^{*}\right)\right)\right)\left(x_{0}\right)-\varepsilon / 4 .
$$

Since $A\left(m_{1}^{*}\right) \subseteq K_{1}$ and $K_{1}$ is determined by time $\tau_{1}$, combining (7.5) and (7.6), we get

$$
\int_{K_{1}} \bar{M}\left(A\left(\left(m_{1}^{*}\right)\right) p_{\tau_{1}}\right)\left(h_{\tau_{1}}\right) d \lambda(h)>\bar{M}(A)\left(x_{0}\right)-\varepsilon / 2 .
$$

Suppose, next, that $K_{1}, K_{2}, \ldots, K_{n}, \tau_{1}, \tau_{2}, \ldots, \tau_{n}, m_{1}^{*}, m_{2}^{*}, \ldots, m_{n}^{*}$, and $\phi_{1}, \phi_{2}, \ldots, \phi_{n-1}$ have been defined. Define $J: H \times \omega^{n} \times N \rightarrow \omega$ as follows:

$$
\begin{aligned}
& J\left(h, m_{1}, m_{2}, \ldots, m_{n}, k\right)=\text { least } m \text { such that } \\
& \bar{M}\left(A\left(\left(m_{1}, m_{2}, \ldots, m_{n}, m\right)\right) h_{1} h_{2} \cdots h_{k}\right)\left(h_{k}\right) \\
& \quad>\bar{M}\left(A\left(\left(m_{1} m_{2}, \ldots, m_{n}\right)\right) h_{1} h_{2} \cdots h_{k}\right)\left(h_{k}\right)-\frac{\varepsilon}{2^{n+2}} .
\end{aligned}
$$

Then $J$ is well defined. To see this, first observe that

$$
\left(A\left(\left(m_{1}, m_{2}, \ldots, m_{n}, m\right)\right) h_{1} h_{2} \cdots h_{k}\right)_{m \in \omega}
$$

is an increasing $\Sigma_{1}^{1}\left(\left\langle h_{1}, h_{2}, \ldots, h_{k}\right\rangle\right)$ sequence whose union is

$$
A\left(\left(m_{1}, m_{2}, \ldots, m_{n}\right)\right) h_{1} h_{2} \cdots h_{k} .
$$

Now use a relativized version of Corollary 6.7 to see that there exists $m$ satisfying (7.7).

It follows easily from Lemma 4.1 that $J$ is analytically measurable. Furthermore,

$h \equiv{ }_{k} h^{\prime} \quad$ implies $\quad J\left(h, m_{1}, m_{2}, \ldots, m_{n}, k\right)=J\left(h^{\prime}, m_{1}, m_{2}, \ldots, m_{n}, k\right)$. 
Now set

$$
m_{n+1}^{*}(h)=J\left(h, m_{1}^{*}, m_{2}^{*}(h), \ldots, m_{n}^{*}(h), \tau_{n}(h)\right), \quad h \in H,
$$

so that $m_{n+1}^{*}$ is universally measurable and determined by time $\tau_{n}$. Next, define $K_{n+1}$ by the formula

$$
\begin{array}{r}
h \in K_{n+1} \leftrightarrow\left(\exists m_{2}\right)\left(\exists m_{3}\right) \cdots\left(\exists m_{n+1}\right) \\
{\left[m_{2}^{*}(h)=m_{2} \& m_{3}^{*}(h)=m_{3} \& \cdots \& m_{n+1}^{*}(h)\right.} \\
=m_{n+1} \&\left(\exists i_{1} \leq m_{1}^{*}\right)\left(\exists i_{2} \leq m_{2}\right) \cdots\left(\exists i_{n+1} \leq m_{n+1}\right) \\
\left.\left(h \in B\left(\left(i_{1}, i_{2}, \ldots, i_{n+1}\right)\right)\right)\right] .
\end{array}
$$

It is easily verified, using the facts that the functions $m_{2}^{*}, m_{3}^{*}, \ldots, m_{n+1}^{*}$ are determined by time $\tau_{n}$ and that $B(s)$ is clopen in $H$, that $K_{n+1}$ is clopen. Furthermore, $K_{n+1}$ is universally measurable, since the functions $m_{2}^{*}, m_{3}^{*}, \ldots$, $m_{n+1}^{*}$ are and the sets $B(s)$ are Borel. Plainly, condition (b) is fulfilled and $K_{n+1}^{n+1} \subseteq K_{n}$.

Fix a function $g_{n+1}: H \times \omega^{n+1} \times N \rightarrow \omega^{\omega}$ having the properties stated in Lemma 7.3. Define $\tau_{n+1}: H \rightarrow N$ by the formula

$$
\tau_{n+1}(h)=\tau_{n}(h)+\left\{g_{n+1}\left(h, m_{1}^{*}, m_{2}^{*}(h), \ldots, m_{n+1}^{*}(h), \tau_{n}(h)\right)\right\}\left(h^{\prime}\right),
$$

where $h^{\prime}=\left(h_{\tau_{n}(h)+1}, h_{\tau_{n}(h)+2}, \ldots\right)$. Using the inductive hypothesis and Lemma 7.3 , it is not hard to verify that $\tau_{n+1}$ is a universally measurable stop rule such that $\tau_{n}<\tau_{n+1}$ and $K_{n+1}$ is determined by time $\tau_{n+1}$.

In order to define $\phi_{n}$, proceed as follows. First, define $\phi: H \times \omega^{n+1} \times N \times H \rightarrow$ $[0,1]$ by

$$
\begin{array}{r}
\phi\left(h, m_{1}, m_{2}, \ldots, m_{n+1}, k, h^{\prime}\right) \\
=\bar{M}\left(A\left(\left(m_{1}, m_{2}, \ldots, m_{n+1}\right)\right) h_{1} h_{2} \ldots h_{k}\right. \\
\left.\quad p_{\left\{g_{n+1}\left(h, m_{1}, m_{2}, \ldots, m_{n+1}, k\right)\right\}}\right)\left(h_{i}^{\prime}\right),
\end{array}
$$

where $i=\left\{g_{n+1}\left(h, m_{1}, m_{2}, \ldots, m_{n+1}, k\right)\right\}\left(h^{\prime}\right)$.

Observe first that, for fixed $m_{1}, m_{2}, \ldots, m_{n+1}, h^{\prime}, \phi$ as a function in $h$ and $k$ is adapted. Next, by doing a calculation similar to that in the proof of Lemma 4.1 and using Lemma 7.3, one verifies easily that $\phi$ is an upper analytic function. So, by a selection theorem [12, Lemma 2.1], there is an analytically measurable function $\psi: H \times \omega^{n+1} \times N \rightarrow P(H)$ such that for every $\left(h, m_{1}, m_{2}, \ldots, m_{n+1}, k\right) \in H \times \omega^{n+1} \times N$,

$$
\psi\left(h, m_{1}, m_{2}, \ldots, m_{n+1}, k\right) \in \Sigma\left(h_{k}\right)
$$

and

$$
\begin{array}{r}
\int \phi\left(h, m_{1}, m_{2}, \ldots, m_{n+1}, k, h^{\prime}\right) \psi\left(h, m_{1}, m_{2}, \ldots, m_{n+1}, k\right)\left(d h^{\prime}\right) \\
>\sup _{\nu \in \Sigma\left(h_{k}\right)} \int \phi\left(h, m_{1}, m_{2}, \ldots, m_{n+1}, k, h^{\prime}\right) \nu\left(d h^{\prime}\right)-\frac{\varepsilon}{2^{n+2}} .
\end{array}
$$

Now an application of a relativized version of Corollary 4.5 to the

$$
\Sigma_{1}^{1}\left(\left\langle h_{1}, h_{2}, \ldots, h_{k}\right\rangle\right)
$$


set $A\left(\left(m_{1}, m_{2}, \ldots, m_{n+1}\right)\right) h_{1} h_{2} \ldots h_{k}$ and the $\Delta_{1}^{1}\left(\left\langle h_{1}, h_{2}, \ldots, h_{k}\right\rangle\right)$-recursive stop rule $\left\{g_{n+1}\left(h, m_{1}, m_{2}, \ldots, m_{n+1}, k\right)\right\}$ yields

$$
\begin{aligned}
\bar{M}\left(A\left(m_{1}, m_{2}, \ldots, m_{n+1}\right) h_{1} h_{2} \cdots h_{k}\right)\left(h_{k}\right) \\
\quad \leq \sup _{\nu \in \Sigma\left(h_{k}\right)} \int \phi\left(h, m_{1}, m_{2}, \ldots, m_{n+1}, k, h^{\prime}\right) \nu\left(d h^{\prime}\right)
\end{aligned}
$$

for every $\left(h, m_{1}, m_{2}, \ldots, m_{n+1}, k\right) \in H \times \omega^{n+1} \times N$.

Define $\phi_{n}: H \times N \rightarrow P(H)$ by setting

$$
\phi_{n}(h, k)=\left\{\begin{array}{l}
\psi\left(\left(h_{1}, h_{2}, \ldots, h_{k}, x^{*}, x^{*}, \ldots\right),\right. \\
\left.\quad m_{1}^{*}, m_{2}^{*}(h), \ldots, m_{n+1}^{*}(h), k\right) \quad \text { if } k \geq \tau_{n}(h), \\
\bar{\psi}\left(h_{k}\right) \quad \text { if } k<\tau_{n}(h),
\end{array}\right.
$$

where $\bar{\psi}: X \rightarrow P(H)$ is an analytically measurable selector for $\Sigma$ regarded as a subset of $X \times P(H)$, and $x^{*}$ is a fixed point in $X$.

Now it is easy to check that $\phi_{n}$ is universally measurable, adapted, and $\phi_{n}(h, k) \in \Sigma\left(h_{k}\right)$ for every $h \in H$ and $k \in N$.

It follows from (7.9)-(7.13) and the property of $\phi$ mentioned immediately after (7.10) that for any $h \in H$,

$$
\begin{gathered}
\int \bar{M}\left(A\left(\left(m_{1}^{*}, m_{2}^{*}(h), \ldots, m_{n+1}^{*}(h)\right)\right) q_{n}(h) p_{\tau_{n+1}\left[q_{n}(h)\right]}\left(h^{\prime}\right)\right) \\
\left(h_{\tau_{n+1}\left[q_{n}(h)\right]}^{\prime}\right) \phi_{n}\left(h, \tau_{n}(h)\right)\left(d h^{\prime}\right) \\
>\bar{M}\left(A\left(\left(m_{1}^{*}, m_{2}^{*}(h), \ldots, m_{n+1}^{*}(h)\right)\right) q_{n}(h)\right)\left(h_{\tau_{n}}\right)-\frac{\varepsilon}{2^{n+2}} \\
>\bar{M}\left(A\left(\left(m_{1}^{*}, m_{2}^{*}(h), \ldots, m_{n}^{*}(h)\right)\right) q_{n}(h)\right)\left(h_{\tau_{n}}\right)-\frac{\varepsilon}{2^{n+1}}
\end{gathered}
$$

where the last inequality is by virtue of (7.7) and (7.8). Condition (d) of Lemma 7.2 now follows from the previous inequality by observing that

$$
A\left(\left(m_{1}^{*}, m_{2}^{*}(h), \ldots, m_{n+1}^{*}(h)\right) q_{n}(h)\right.
$$

is contained in $K_{n+1} q_{n}(h)$ and that $K_{n+1} q_{n}(h)$ is determined by time $\tau_{n+1}\left[q_{n}(h)\right]$. This completes the proof of Theorem 7.1.

\section{Proof of Theorem 1.1}

It is now an easy matter to deduce Theorem 1.1 from Theorem 7.1. So let us return to the setting of $\S 1$. First, assume that $X=2^{\omega}, \Gamma$ is an analytic gambling house on $X$, and $A$ is an analytic subset of $H=X \times X \times \cdots$. For each $x \in X$, let $\Sigma(x)$ denote the set of probability measures on the Borel sets of $H$ induced by universally measurable strategies available at $x$. It was observed by Dellacherie [3] (see also Sudderth [23]) that $\Sigma$ is an analytic subset of $X \times P(H)$. Furthermore, $\Sigma$ is a global gambling house of the type that was considered in $\S \S 3-7$. By a result in [15, p. 160], we can choose $\alpha \in \omega^{\omega}$ (indeed, $\alpha$ can be chosen in $2^{\omega}$ ) such that both $A$ and $\Sigma$ are $\Sigma_{1}^{1}(\alpha)$ sets. Since the global gambling house $\Sigma$ is $\Sigma_{1}^{1}(\alpha)$, we modify the set function $\bar{M}$ introduced 
in (4.2). Define a new set function $\overline{\bar{M}}$ as follows:

$$
\begin{aligned}
& \overline{\bar{M}}\left(E ; x_{1}, x_{2}, \ldots, x_{k} ; x\right) \\
& \quad=\inf \left\{M(O)(x): O \text { is a } \Delta_{1}^{1}\left(\left\langle\alpha, x_{1}, x_{2}, \ldots, x_{k}, x\right\rangle\right),\right. \\
&\text { open set containing } \left.E x_{1} x_{2} \cdots x_{k}\right\}
\end{aligned}
$$

for any $\Sigma_{1}^{1}(\alpha)$ set $E \subseteq H$ and $x_{1}, x_{2}, \ldots, x_{k}, x \in X$. Abbreviate the left side of $(8.1)$ by $\overline{\bar{M}}\left(E x_{1}, x_{2} \cdots x_{k}\right)(x)$.

Relativize Theorem 7.1 to $\alpha$. This yields

$$
\overline{\bar{M}}(A)(x)=M(A)(x)
$$

for every $x \in X$. But

$$
M^{*}(A)(x) \leq \overline{\bar{M}}(A)(x), \quad x \in X,
$$

since a $\Delta_{1}^{1}(\langle\alpha, x\rangle)$ set is Borel. So

$$
M^{*}(A)(x) \leq M(A)(x)
$$

for every $x \in X$. The inequality in the reverse direction being obvious, we have proved Theorem 1.1 when $X=2^{\omega}$.

To complete the proof, suppose that $X$ is a Borel (or even analytic) subset of a Polish space. By the Borel isomorphism theorem, we may regard $X$ without loss of generality as an analytic subset of $2^{\omega}$ and also suppose that $X \neq 2^{\omega}$. Identify each element $\mu$ of $P(X)$ with the unique element of $P\left(2^{\omega}\right)$ whose trace on $X$ is $\mu$. It is now easy to see that $P(X)$ is (can be identified with) an analytic subset of $P\left(2^{\omega}\right)$ and hence that $\Gamma$ is an analytic subset of $2^{\omega} \times P\left(2^{\omega}\right)$. Fix $x^{*} \in 2^{\omega}-X$ and define a new gambling house $\widetilde{\Gamma}$ on $2^{\omega}$ as follows:

$$
\widetilde{\Gamma}(x)= \begin{cases}\Gamma(x) \cup\left\{\delta\left(x^{*}\right)\right\} & \text { if } x \in X, \\ \left\{\delta\left(x^{*}\right)\right\} & \text { if } x \in 2^{\omega}-X .\end{cases}
$$

Since the set $\Gamma_{0}=\left\{\left(x, \delta\left(x^{*}\right)\right) \in 2^{\omega} \times P\left(2^{\omega}\right): x \in 2^{\omega}\right\}$ is Borel in $2^{\omega} \times P\left(2^{\omega}\right)$ and $\widetilde{\Gamma}=\Gamma \cup \Gamma_{0}$, it follows that $\widetilde{\Gamma}$ is an analytic subset of $2^{\omega} \times P\left(2^{\omega}\right)$. Hence $\widetilde{\Gamma}$ is an analytic gambling house on $2^{\omega}$. Let $\widetilde{M}$ denote the optimal reward operator associated with $\widetilde{\Gamma}$ and let $\widetilde{H}=2^{\omega} \times 2^{\omega} \times \cdots$. Let $A$ be an analytic subset of $H=X \times X \times \cdots, x \in X$, and $\varepsilon>0$. Plainly, $A$ is analytic in $\tilde{H}$. So by the result established for $2^{\omega}$ in the previous paragraph, there is a Borel, open subset $O^{\prime}$ of $\widetilde{H}$ such that $O^{\prime}$ contains $A$ and

$$
\widetilde{M}\left(O^{\prime}\right)\left(x^{\prime}\right)<\widetilde{M}(A)(x)+\varepsilon .
$$

Let $O=O^{\prime} \cap H$, so $O$ is Borel and open in $H$ and contains $A$. Since $A \subseteq H$ and $x \in X$ it is easy to see that $\widetilde{M}(A)(x)=M(A)(x)$. Hence,

$$
M(O)(x) \leq \widetilde{M}\left(O^{\prime}\right)(x)<M(A)(x)+\varepsilon .
$$

This completes the proof of Theorem 1.1.

We conclude this section with the observation that Theorem 1.1 implies a genuine "capacitability" result for the set functions $M^{*}(\cdot)(x)$, even though $M^{*}(\cdot)(x)$ need not be a capacity [13]. 
Theorem 8.1. For every $x \in X$ and analytic subset $A$ of $H$,

$$
M^{*}(A)(x)=\sup \left\{M^{*}(K)(x): K \subseteq A \& K \text { is } T_{1} \text {-compact }\right\} .
$$

Proof. Since $M^{*}$ is monotone, only the equation $\leq$ needs to be established. This follows from Theorem 1.1 and the following string of inequalities:

$$
\begin{aligned}
M(A)(x) & =\sup _{\sigma \in \Sigma(x)} \sigma(A) \\
& =\sup _{\sigma \in \Sigma(x)} \sup \left\{\sigma(K): K \subseteq A \& K \text { is } T_{1} \text {-compact }\right\} \\
& =\sup \sup _{\sigma \in \Sigma(x)}\left\{\sigma(K): K \subseteq A \& K \text { is } T_{1} \text {-compact }\right\} \\
& =\sup \left\{M(K)(x): K \subseteq A \& K \text { is } T_{1} \text {-compact }\right\} \\
& \leq \sup \left\{M^{*}(K)(x): K \subseteq A \& K \text { is } T_{1} \text {-compact }\right\} .
\end{aligned}
$$

\section{ADEQUACY OF MEASURABLE STRATEgIES}

The gambling theory of Dubins and Savage [4] takes place in a very general finitely additive framework in which a player is permitted to use nonmeasurable strategies. An interesting question, which was posed by Dubins and Savage, is whether a player can do better if allowed to use nonmeasurable strategies or whether, to the contrary, measurable strategies are adequate. The main result of this section is that for a wide class of gambling problems nonmeasurable strategies do not give the gambler any advantage.

Suppose that $\Gamma$ is a gambling house on a Borel subset $X$ of a Polish space. Assume that every gamble $\gamma$ available in $\Gamma$ is defined on all subsets of $X$ as a finitely additive probability measure, whose restriction on the Borel subsets of $X$ is countably additive. Identifying each gamble with its restriction to the Borel sets of $X$, we can regard $\Gamma$ as a subset of $X \times P(X)$. As in $\S 1$, we will assume that $\Gamma$ is an analytic subset of $X \times P(X)$.

Given an analytically measurable set $A \subseteq H$, we can evaluate $\sigma(A)$ for strategies $\sigma$ which are not necessarily measurable by using the Dubins and Savage definition of the measure $\sigma$ as extended by Purves and Sudderth [18, Theorem 5.3]. If the strategy $\sigma$ is measurable, and $A$ is as above, then $\sigma(A)$ has the same value when calculated in either setting.

We will denote the optimal reward operator when both measurable and nonmasurable strategies are allowed by $\Gamma$.

Theorem 9.1. If $X$ is a Borel subset of a Polish space, $\Gamma$ an analytic gambling house on $X$, and $A$ an analytic subset of $H=X \times X \times \cdots$, then $\Gamma(A)=M(A)$.

Proof. It was observed in the course of the proof of Theorem 7.2 of [12] that $M(O)=\Gamma(O)$ for any Borel, open set $O$. It therefore follows from Theorem 1.1 that, for any $x \in X$,

$$
\begin{aligned}
M(A)(x) & =\inf \{M(O)(x): O \supseteq A \& O \text { is Borel, open }\} \\
& \geq \inf \{\Gamma(O)(x): O \supseteq A \& O \text { is open }\} \geq \Gamma(A)(x) .
\end{aligned}
$$

Since the inequality in the opposite direction is trivial, the theorem is proved.

The rest of this section will be devoted to the extension of Theorem 9.1 to bounded, upper analytic functions on $H$ (Theorem 1.2). The proof uses ideas from Monticino [14] (see, in particular, Theorem 4.2 in [14]). 
As in Theorem 1.2, assume that $g$ is a bounded, upper analytic function on $H$. For any strategy $\sigma$, measurable or not, $\sigma g=\int g d \sigma$ is well defined, since $g$ is analytically measurable. For the same reason, if $\sigma$ is measurable, then the value of $\sigma g$, whether computed in the finitely additive or countably additive mode, is the same.

The first step in the proof of Theorem 1.2 is the uniform approximation of $g$ by an upper analytic function with only finitely many values. Assume without loss of generality that $0 \leq g \leq 1$ and fix $\varepsilon>0$. Let $n(\varepsilon)$ be the least integer $n$ such that $n \varepsilon \geq 1$ and, for $n=1,2, \ldots, n(\varepsilon)$, define $A^{n}=\{h \in H: g(h) \geq$ $n \varepsilon\}$. Then set

$$
\tilde{g}=\varepsilon\left[A^{1}+A^{2}+\cdots+A^{n(\varepsilon)}\right],
$$

where we are denoting the indicator function of the set $A^{i}$ by the symbol $A^{i}$. The $A^{i}$ are analytic. Thus $\tilde{g}$ is upper analytic with values in $\{0, \varepsilon, 2 \varepsilon, \ldots$, $n(\varepsilon) \varepsilon\}$. Moreover, $\tilde{g} \leq g \leq \tilde{g}+\varepsilon$.

Thus, it suffices to prove Theorem 1.2 for $\tilde{g}$ and, consequently, for $\varepsilon^{-1} \tilde{g}$. So, without loss of generality, assume from now on that

$$
g=A^{1}+A^{2}+\cdots+A^{n},
$$

where $A^{1} \supseteq A^{2} \supseteq \cdots \supseteq A^{n}$ are analytic subsets of $H$.

The next step is an approximation of each analytic set $A$ by an anatic, $G_{\delta}$ set $G$ as was essentially done in $\S 6$. Fix the analytic set $A \subseteq H$ and let $0<\varepsilon<1$.

Define

$$
B=B_{\varepsilon}(A)=\left\{p \in X^{*}: \Gamma(A p)(l(p))>1-\varepsilon\right\},
$$

where $l(p)$ is the last coordinate of the finite sequence $p$ and $X^{*}=\bigcup_{m \in N} X^{m}$. By virtue of Theorem 9.1 , the set $B$ would be the same if we replaced $\Gamma$ by $M$ in its definition. Consequently, $B$ is an analytic subset of $X^{*}$ (see the proof of Lemma 6.3). Next define the analytic $G_{\delta}$ set

$$
G=G_{\varepsilon}(A)=\left\{h \in H: p_{n}(h) \in B \text { infinitely often }\right\} .
$$

Lemma 9.2. For all $x \in X, \Gamma(A \cap(H-G))(x)=0$.

Proof. Suppose, by way of contradiction, that, for some $x$ and $\sigma$ available at $x$,

$$
\sigma(A \cap(H-G))>0 .
$$

By the finitely additive Levy 0-1 law [20],

$$
\sigma\left[p_{n}(h)\right]\left((A \cap(H-G)) p_{n}(h)\right) \rightarrow 1
$$

for $\sigma$-almost every $h \in A \cap(H-G)$. Hence, there is an $h \in H-G$ such that $\sigma\left[p_{n}(h)\right]\left(A p_{n}(h)\right)>1-\varepsilon$ for infinitely many $n$. Since $\Gamma\left(A p_{n}(h)\right)\left(h_{n}\right) \geq$ $\sigma\left[p_{n}(h)\right]\left(A p_{n}(h)\right), h \in G$, which yields the desired contradiction.

Now, for each $A^{i}$ occurring in (9.1), define

$$
B_{i}=B_{\varepsilon}\left(A^{i}\right), \quad G_{i}=G_{\varepsilon}\left(A^{i}\right) .
$$

Notice that $B_{1} \supseteq B_{2} \supseteq \cdots \supseteq B_{n}$ and $G_{1} \supseteq G_{2} \supseteq \cdots \supseteq G_{n}$. Now define $g_{\varepsilon}$ on $H$ and $u_{\varepsilon}$ on $X^{*}$ by

$$
g_{\varepsilon}=G_{1}+G_{2}+\cdots+G_{n}, \quad u_{\varepsilon}=B_{1}+B_{2}+\cdots+B_{n} .
$$


We note that both $g_{\varepsilon}$ and $u_{\varepsilon}$ are upper analytic functions. Finally, define, for $x \in X$,

$$
\left(T u_{\varepsilon}\right)(x)=\sup \left\{\int u_{\varepsilon}\left(p_{t}\right) d \sigma: \sigma \text { available at } x, t \text { a stop rule }\right\} .
$$

The notation $T u_{\varepsilon}$ is consistent with that in [5] if we pass to the "partial history house." In particular, the result of Strauch [22], as modified in [5, Lemma 4.2], implies that $\left(T u_{\varepsilon}\right)(x)$ would have the same value if the supremum were taken only over measurable $\sigma$ available at $x$ and Borel stop rules $t$.

The final steps of the argument will show that

$$
\Gamma g \leq \Gamma g_{\varepsilon} \leq T u_{\varepsilon} \leq M g+(n+1) \varepsilon .
$$

This will suffice to complete the proof of Theorem 1.2 since $\varepsilon$ is arbitrary, $n$ is fixed, and, obviously, $M g \leq \Gamma g$.

Lemma 9.3. $\Gamma g \leq \Gamma g_{\varepsilon}$.

Proof. Let $x \in X$ and $\sigma$ be available at $x$. Then

$$
\begin{aligned}
\sigma g & =\sigma\left(A^{1}\right)+\sigma\left(A^{2}\right)+\cdots+\sigma\left(A^{n}\right) \\
& \leq \sigma\left(G_{1}\right)+\sigma\left(G_{2}\right)+\cdots+\sigma\left(G_{n}\right)=\sigma g_{\varepsilon},
\end{aligned}
$$

where the inequality holds because, for each $i, \sigma\left(A^{i} \cap\left(H-G_{i}\right)\right)=0$ by Lemma 9.2.

Lemma 9.4. $\Gamma g_{\varepsilon} \leq T u_{\varepsilon}$.

Proof. First notice that, for each $h$,

$$
\begin{aligned}
g_{\varepsilon}(h) & =G_{1}(h)+G_{2}(h)+\cdots+G_{n}(h) \\
& =\varlimsup_{k} B_{1}\left(p_{k}(h)\right)+\varlimsup_{k} B_{2}\left(p_{k}(h)\right)+\cdots+\varlimsup_{k} B_{n}\left(p_{k}(h)\right) \\
& =\varlimsup_{k}\left(B_{1}+B_{2}+\cdots+B_{n}\right)\left(p_{k}(h)\right) .
\end{aligned}
$$

To check the final equality, recall that $B_{1} \supseteq B_{2} \supseteq \cdots \supseteq B_{n}$ so that $g_{\varepsilon}(h)=m$ if and only if $\varlimsup_{k} B_{1}\left(p_{k}(h)\right)=\cdots=\varlimsup_{\overline{\lim }} B_{m}\left(p_{k}(h)\right)=1$ and $\overline{\lim }_{k} B_{m+1}\left(p_{k}(h)\right)=$ $\cdots=\overline{\lim }_{k} B_{n}\left(p_{k}(h)\right)=0$ for $m=0,1, \ldots, n$.

Now, for any $\sigma$ available at $x$,

$$
\begin{aligned}
\sigma g_{\varepsilon} & =\int \varlimsup_{k}\left(B_{1}+B_{2}+\cdots+B_{n}\right)\left(p_{k}\right) d \sigma=\int \varlimsup_{k} u_{\varepsilon}\left(p_{k}\right) d \sigma \\
& =\varlimsup_{t} \int u_{\varepsilon}\left(p_{t}\right) d \sigma \leq \sup _{t} \int u_{\varepsilon}\left(p_{t}\right) d \sigma .
\end{aligned}
$$

Here the final equality is a trivial variation of the Fatou equation [17, Theorem 10.4] (see also [24] for a proof of a countably additive version of the Fatou equation; this proof can be adapted to the finitely additive setting by using the finitely additive Levy $0-1$ Law [20]). Take the supreumum over $\sigma$ available at $x$ to finish the proof.

Lemma 9.5. $T u_{\varepsilon} \leq M g+(n+1) \varepsilon$.

Proof. Fix $x \in X$. By virtue of the remark immediately following the definition of $T u_{\varepsilon}$, we can choose a measurable $\hat{\sigma}$ available at $x$ and a Borel measurable stop rule $t$ such that

$$
\int u_{\varepsilon}\left(p_{t}\right) d \hat{\sigma}>T u_{\varepsilon}(x)-\varepsilon
$$


Next we use a selection theorem [12, Lemma 2.1] to obtain analytically measurable mappings $\bar{\sigma}_{k}: X^{*} \rightarrow P(H)$ such that $\bar{\sigma}_{k}(p) \in \Sigma(l(p))$ and, if $p \in B_{k}$, then

$$
\bar{\sigma}_{k}(p)\left(A^{k} p\right)>1-\varepsilon
$$

for $k=1,2, \ldots, n$. Then define, for $p \in X^{*}$,

$$
\begin{aligned}
\bar{\sigma}(p) & =\bar{\sigma}_{n}(p) & & \text { if } p \in B_{n}, \\
& =\bar{\sigma}_{n-1}(p) & & \text { if } p \in B_{n-1}-B_{n}, \\
& \vdots & & \\
& =\bar{\sigma}_{1}(p) & & \text { if } p \in B_{1}-B_{2}, \\
& =\bar{\sigma}_{1}(p) & & \text { (say) elsewhere. }
\end{aligned}
$$

Then $\bar{\sigma}$ is analytically measurable from $X^{*}$ to $P(H)$. Furthermore, since $A^{1} \supseteq A^{2} \supseteq \cdots \supseteq A^{n}$, it follows that

$$
\bar{\sigma}(p)\left(A^{k} p\right)>1-\varepsilon \quad \text { if } p \in B_{k}
$$

for $k=1,2, \ldots, n$. Consequently,

$$
\begin{aligned}
\bar{\sigma}\left(p_{t}\right)\left(g p_{t}\right) & =\bar{\sigma}\left(p_{t}\right)\left(\left(A^{1}+A^{2}+\cdots+A^{n}\right) p_{t}\right) \\
& \geq\left(B_{1}+B_{2}+\cdots+B_{n}\right)\left(p_{t}\right)-n \varepsilon=u_{\varepsilon}\left(p_{t}\right)-n \varepsilon .
\end{aligned}
$$

Now let $\sigma$ be the measurable strategy available at $x$ which agrees with $\hat{\sigma}$ prior to time $t$ and satisfies $\sigma\left[p_{t}(h)\right]=\bar{\sigma}\left(p_{t}(h)\right)$ for $\sigma$-almost all $h$. Then

$$
\begin{aligned}
M g(x) & \geq \sigma g=\int \bar{\sigma}\left(p_{t}\right)\left(g p_{t}\right) d \hat{\sigma} \\
& \geq \int u_{\varepsilon}\left(p_{t}\right) d \hat{\sigma}-n \varepsilon>T u_{\varepsilon}(x)-(n+1) \varepsilon .
\end{aligned}
$$

We remark that it is consistent with ZFC that Theorem 9.1 does not extend to coanalytic sets. Indeed, under the assumption that there exists a non-Lebesgue measurable PCA subset of $[0,1]$, Monticino [14] constructed a Borel gambling house $\Gamma$ on a Polish space such that there is a coanalytic set $C \subseteq H$ with $\Gamma(C) \neq M(C)$. In other words, for some initial fortune $x$, nonmeasurable strategies provide a distinct advantage over measurable strategies. It also follows that $M^{*}(C)(x)>M(C)(x)$ for some $x \in X$.

\section{ApProximating FUnCtions}

In this final section we prove an approximation result for bounded, upper analytic functions on the set $H$ of histories, which is analogous to the result for sets proved in Theorem 1.1.

A subset of $H$ which is a countable intersection of Borel, open subsets of $H$ will be called a special $G_{\delta}$ set. The following theorem is the approximation result for functions.

Theorem 10.1. Let $\Gamma$ be an analytic gambling house on a Borel subset $X$ of a Polish space. If $g$ is a bounded, upper analytic function on $H$, then

$$
(M g)(x)=\inf (M f)(x),
$$


where the infimum is over all functions $f: H \rightarrow \mathfrak{R}$ such that $f \geq g, f$ takes on finitely many values, and $\{f \geq c\}$ is a special $G_{\delta}$ for every $c \in \mathfrak{R}$.

Proof. It follows by virtue of the arguments of the previous section that it suffices to prove the theorem when $g$ is given by (9.1). Fix $\varepsilon>0$ and $x_{0} \in$ $X$. Let the sets $G_{i}$ be defined by (9.2). Recall that each $G_{i}$ is a countable intersection of analytic, open sets, and that, by virtue of Lemma 9.2,

$$
M\left(A^{i} \cap\left(H-G_{i}\right)\right)(x)=0 \quad \text { for all } x \in X .
$$

We now need a lemma.

Lemma 10.2. There exist special $G_{\delta}$ sets $\widetilde{G}_{i}, i=1,2, \ldots, n$, with the following properties:

(a) $\widetilde{G}_{1} \supseteq \widetilde{G}_{2} \supseteq \cdots \supseteq \widetilde{G}_{n}$,

(b) $G_{i} \subseteq \widetilde{G}_{i}, i=1,2, \ldots, n$, and

(c) $M\left(\widetilde{\widetilde{G}}_{1}+\widetilde{G}_{2}+\cdots+\widetilde{G}_{n}\right)\left(x_{0}\right) \leq M\left(G_{1}+G_{2}+\cdots+G_{n}\right)\left(x_{0}\right)+\varepsilon$.

Proof. Let

$$
D=\left\{(h, a) \in H \times[0, n]: G_{1}(h)+G_{2}(h)+\cdots+G_{n}(h) \geq a\right\} .
$$

Plainly, $D$ is analytic in $H \times[0, n]$. So, By Corollary 4.4 in [12], there is a Borel subset $E$ of $H \times[0, n]$ such that $D \subseteq E$ and

$$
\sup _{\sigma \in \Sigma\left(x_{0}\right)}(\sigma \times \lambda)(E) \leq \sup _{\sigma \in \Sigma\left(x_{0}\right)}(\sigma \times \lambda)(D)+\varepsilon,
$$

where $\lambda$ is Lebesgue measure on $[0, n]$. Set $\phi(h)=\lambda\left(E_{h}\right), h \in H$. Clearly, then, $\phi$ is Borel measurable, $\phi \geq G_{1}+G_{2}+\cdots+G_{n}$, and, because, of (10.1),

$$
(M \phi)\left(x_{0}\right) \leq M\left(G_{1}+G_{2}+\cdots+G_{n}\right)\left(x_{0}\right)+\varepsilon .
$$

Let $C_{i}=\{\phi \geq i\}, i=1,2, \ldots, n$, so $C_{i} \supseteq G_{i}$. Hence, by the proof of Corollary 6.8 in [12], there is a special $G_{\delta}$ set $F_{i}$ such that $C_{i} \supseteq F_{i} \supseteq G_{i}$. Let $\widetilde{G}_{i}=F_{1} \cap F_{2} \cap \cdots \cap F_{i}, i=1,2, \ldots, n$. Then it is straightforward to verify that the sets $\widetilde{G}_{i}$ satisfy conditions (a)-(c) of the lemma.

To continue with the proof of Theorem 10.1 , let $\widetilde{G}_{1}, \widetilde{G}_{2}, \ldots, \widetilde{G}_{n}$ be as in Lemma 10.2. It follows that $A^{i} \cap\left(H-\widetilde{G}_{i}\right) \subseteq A^{i} \cap\left(H-G_{i}\right)$ so that

$$
M\left(A^{i} \cap\left(H-\widetilde{G}_{i}\right)\right)\left(x_{0}\right)=0 .
$$

Since $A^{i} \cap\left(H-\widetilde{G}_{i}\right)$ is analytic, we can apply Theorem 1.1 to get a Borel, open set $O_{i}^{\prime}$ containing $A^{i} \cap\left(H-\widetilde{G}_{i}\right)$ such that

$$
M\left(O_{i}^{\prime}\right)\left(x_{0}\right)<\varepsilon / n^{2}, \quad i=1,2, \ldots, n .
$$

Set $O_{i}=O_{i}^{\prime} \cup O_{i+1}^{\prime} \cup \cdots \cup O_{n}^{\prime}$, so $O_{i}$ is Borel, open, $O_{1} \supseteq O_{2} \supseteq \cdots \supseteq O_{n}$ and, by $[13$, Lemma 2.1$]$,

$$
\begin{aligned}
M\left(O_{i}\right)\left(x_{0}\right) \leq M\left(O_{i}^{\prime}\right)\left(x_{0}\right)+M\left(O_{i+1}^{\prime}\right)\left(x_{0}\right)+\cdots+M\left(O_{n}^{\prime}\right)\left(x_{0}\right)<\varepsilon / n, & \\
i & =1, \ldots, n .
\end{aligned}
$$

Define

$$
f=\widetilde{G}_{1}+\widetilde{G}_{2}+\cdots+\widetilde{G}_{n}+O_{1}+O_{2}+\cdots+O_{n} .
$$


Since $A^{i} \subseteq \widetilde{G}_{i} \cup O_{i}$, it follows that $f \geq g$. Clearly, $f$ takes on finitely many values and $\{f \geq c\}$ is a special $G_{\delta}$ for every $c \in \mathfrak{R}$. Finally, for any $\sigma \in \Sigma\left(x_{0}\right)$,

$$
\begin{aligned}
\sigma f & =\sigma\left(\widetilde{G}_{1}+\widetilde{G}_{2}+\cdots+\widetilde{G}_{n}\right)+\sigma\left(O_{1}\right)+\sigma\left(O_{2}\right)+\cdots+\sigma\left(O_{n}\right) \\
& \leq \sigma\left(\widetilde{G}_{1}+\widetilde{G}_{2}+\cdots+\widetilde{G}_{n}\right)+M\left(O_{1}\right)\left(x_{0}\right)+M\left(O_{2}\right)\left(x_{0}\right)+\cdots+M\left(O_{n}\right)\left(x_{0}\right) \\
& \leq \sigma\left(\widetilde{G}_{1}+\widetilde{G}_{2}+\cdots+\widetilde{G}_{n}\right)+\varepsilon .
\end{aligned}
$$

Now take the sup over $\sigma \in \Sigma\left(x_{0}\right)$ to get

$$
\begin{aligned}
(M f)\left(x_{0}\right) & \leq M\left(\widetilde{G}_{1}+\widetilde{G}_{2}+\cdots+\widetilde{G}_{n}\right)\left(x_{0}\right)+\varepsilon \\
& \leq M\left(G_{1}+G_{2}+\cdots+G_{n}\right)\left(x_{0}\right)+2 \varepsilon \leq(M g)\left(x_{0}\right)+(n+3) \varepsilon .
\end{aligned}
$$

where the second inequality is by Lemma 10.2 and the final equality is by virtue of Lemmas 9.4 and 9.5. Since $n$ is fixed and $\varepsilon$ is arbitrary, this completes the proof of Theorem 10.1.

According to Theorem 1.1, for any analytic subset $A$ of $H$ and $x \in X$, $M(A)(x)$ can be approximated by $M(O)(x)$, where $O$ is a Borel, open set containing $A$. It would be reasonable to conjecture from this that if $g$ is a bounded, upper analytic function on $H$, then $(M g)(x)$ can be approximated by $(M f)(x)$, where $f \geq g$ and $\{f \geq c\}$ is a Borel, open set for every $c \in \mathfrak{R}$. We conclude with an example which shows that this conjecture is false.

Example. Let $X=\{0,1,2,3\}, \Gamma(0)=\{\delta(1)\}, \Gamma(2)=\{\delta(2)\}, \Gamma(3)=$ $\{\delta(3)\}$, and $\Gamma(1)=\left\{\delta(1), \frac{2}{3} \delta(2)+\frac{1}{3} \delta(3)\right\}$, where $\delta(x)$ denotes point mass at $x$. Let $G_{i}=\left\{h \in H: h_{n}=i\right.$ infinitely often $\}, i=1,2$. Let $g=$ $G_{1}+2\left(G_{2}-G_{1}\right)$. It is easy to see that $(M g)(0)=\frac{4}{3}$. Now let $f$ be a function on $H$ such that $f \geq g$ and $\{f \geq c\}$ is open for every $c \in \mathfrak{R}$. Then the set $\{f \geq 1\}$ is open and contains $G_{1}$. Let $\tau$ be a stopping time such that $\{f \geq 1\}=\{\tau<\infty\}$. Consider now the history $h=(1,1, \ldots)$. Then $h \in G_{1}$, so $\tau(h)$ is finite. Suppose that $\tau(h)=n$. Define a strategy $\sigma$ available at 0 as follows:

$$
\sigma_{0}=\delta(1),
$$

and for $i \geq 1$,

$$
\sigma_{i}\left(x_{1}, x_{2}, \ldots, x_{i}\right)= \begin{cases}\delta(1) & \text { if }\left(x_{i}=0\right) \text { or }\left(x_{i}=1 \& i \neq n\right), \\ \delta\left(x_{i}\right) & \text { if } x_{i}=2 \text { or } 3 \\ \frac{2}{3} \delta(2)+\frac{1}{3} \delta(3) & \text { if } x_{i}=1 \& i=n\end{cases}
$$

It is now easy to see that $\sigma(\{f \geq 1\})=1$ and $\sigma(\{f \geq 2\}) \geq \sigma\left(G_{2}-G_{1}\right)=\frac{2}{3}$. Consequently,

$$
(M f)(0) \geq \sigma f \geq \sigma(\{f \geq 1\})+\sigma(\{f \geq 2\}) \geq \frac{5}{3} .
$$

\section{REFERENCES}

1. D. P. Bertsekas and S. E. Shreve, Stochastic optimal control: The discrete time case, Academic Press, New York, 1978.

2. G. Choquet, Lectures on analysis, Vol. 1, Integration and Topological Vector Spaces, Benjamin, New York and Amsterdam, 1969. 
3. C. Dellacherie, Quelques résultats sur les maisons de jeux analytiques, (Séminaire de Probabilités XIX, Strasbourg 1983-84), Lecture Notes in Math., vol. 1123, Springer-Verlag, Berlin and New York, 1985, pp. 222-229.

4. L. E. Dubins and L. J. Savage, Inequalities for stochastic processes, Dover, New York, 1976.

5. L. E. Dubins, A. Maitra, R. Purves, and W. Sudderth, Measurable, nonleavable gambling problems, Israel J. Math. 67 (1989), 257-271.

6. A. S. Kechris, Measure and category in effective descriptive set theory, Ann. Math. Logic 5 (1973), 337-384.

7. A. Louveau, Recursiveness and compactness, Higher Set Theory (Proceedings, Oberwolfach, Germany, 1977), Lecture Notes in Math., vol. 669, Springer-Verlag, Berlin and New York, 1978, pp. 303-337.

8. __ Ensembles analytiques et boreliens dans les espaces produits, Astérisque 78 (1980), 1-84.

9. __ Recursivity and capacity theory, Proc. Sympos. Pure Math., vol. 42, Amer. Math. Soc., Providence, R.I., 1985, pp. 285-301.

10. A. Maitra, V. Pestien, and S. Ramakrishnan, Domination by Borel stopping times and some separation properties, Fund. Math. 135 (1990), 189-201.

11. A. Maitra, R. Purves, and W. Sudderth, Leavable gambling problems with unbounded utilities, Trans. Amer. Math. Soc. 320 (1990), 543-567.

12. _ A Borel measurable version of König's Lemma for random paths, Ann. Probab. 19 (1991), 423-451.

13. __ Regularity of the optimal reward operator, University of Minnesota School of Statistics, Tech. Report No. 534, 1989.

14. M. Monticino, The adequacy of universal strategies in analytic gambling problems, Math. Oper. Res. 16 (1991), 21-41.

15. Y. N. Moschovakis, Descriptive set theory, North-Holland, Amsterdam, 1980.

16. K. R. Parthasarathy, Probability measures on metric spaces, Academic Press, New York, 1967.

17. R. Purves and W. Sudderth, Some finitely additive probability, University of Minnesota School of Statistics, Tech. Report No. 220, 1973.

18. __ Some finitely additive probability, Ann. Probab. 4 (1976), 259-276.

19. __ How to stay in a set or König's Lemma for random paths, Israel J. Math. 43 (1982), 139-153.

20. ___ Finitely additive zero-one laws, Sankhyā Ser. A 45 (1983), 32-37.

21. S. Saks, Theory of the integral, Dover, New York, 1964.

22. R. E. Strauch, Measurable gambling houses, Trans. Amer. Math. Soc. 126 (1967), 64-72 (Correction 130 (1968), 184).

23. W. Sudderth, On the existence of good stationary strategies, Trans. Amer. Math. Soc. 135 (1969), 399-414.

24. __ On measurable gambling problems, Ann. Math. Statist. 42 (1971), 260-269.

School of Statistics, University of Minnesota, Minneapolis, Minnesota 55455

Department of Statistics, University of California, Berkeley, California 94720

School of Statistics, University of Minnesota, Minneapolis, Minnesota 55455 